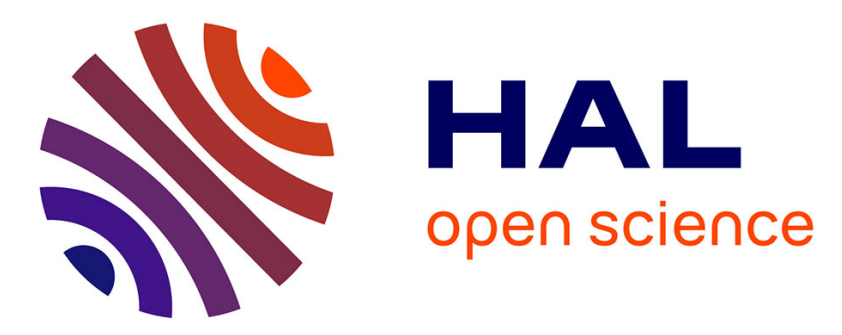

\title{
Optimisation du dimensionnement des alimentations des machines à réluctance variable
}

\author{
Bernard Multon, C. Glaize
}

\section{To cite this version:}

Bernard Multon, C. Glaize. Optimisation du dimensionnement des alimentations des machines à réluctance variable. Revue de Physique Appliquée, 1987, 22 (5), pp.339-357. 10.1051/rphysap:01987002205033900 . jpa-00245549

\section{HAL Id: jpa-00245549 https://hal.science/jpa-00245549}

Submitted on 1 Jan 1987

HAL is a multi-disciplinary open access archive for the deposit and dissemination of scientific research documents, whether they are published or not. The documents may come from teaching and research institutions in France or abroad, or from public or private research centers.
L'archive ouverte pluridisciplinaire HAL, est destinée au dépôt et à la diffusion de documents scientifiques de niveau recherche, publiés ou non, émanant des établissements d'enseignement et de recherche français ou étrangers, des laboratoires publics ou privés. 


\title{
Optimisation du dimensionnement des alimentations des machines à réluctance variable
}

\author{
B. Multon et C. Glaize \\ L.E.SI.R.-E.N.S. de Cachan, 61, avenue du Président-Wilson, 94230 Cachan, France
}

(Reçu le 11 juillet 1986, accepté le 5 février 1987)

\begin{abstract}
Résumé. - Cet article constitue la suite de travaux sur la recherche des formes optimales du courant d'alimentation des machines à réluctance variable pour des critères de maximisation du couple moyen [1]. Nous recherchons maintenant les formes optimales qui minimisent le dimensionnement du convertisseur tant en courant qu'en tension. Après un rappel des différents modes d'alimentation des machines à réluctance variable, nous définissons de nouveaux facteurs de dimensionnement des convertisseurs prenant en compte les valeurs efficaces ou maximales des courant et tension. Nous recherchons ensuite quelles sont les formes d'onde de tension et courant optimales qui minimisent ces facteurs de dimensionnement. Enfin, nous étudions les conséquences de ces ondes d'alimentation sur le couple instantané de la machine éventuellement polyphasée. Nous en déduisons des ensembles alimentations-machines intéressants. Cette étude ne porte que sur des machines monophasées ou «multimonophasées", c'est-à-dire sans couplage magnétique entre phase. Le modèle prend en compte la saturation magnétique et les harmoniques de l'onde spatiale de perméance.
\end{abstract}

\begin{abstract}
This paper follows up the research work conducted on the optimal forms of feeding current in reluctance machine to maximize the mean torque. We now propose to determine the optimal waves minimizing the convector size both in current and voltage. After recording the various feeding modes of reluctance machine, we define new size-power ratios (S.P.R.) which take into account RMS and peak values of current and voltage. We then determine the optimal waves of current and voltage which minimize these ratios. Finally, we study the consequences of these waves on the instantaneous torque. We deduce the notable supply and machine couples. This study deals only with one phase or multistack (without magnetic coupling between the phases) machines. The model considers the magnetic saturation and the harmonics of the spatial waves of permeance.
\end{abstract}

\section{Introduction.}

Les machines à réluctance variable présentent intrinsèquement des avantages indéniables tels que de forts couples massiques, une grande robustesse du rotor, qui les destinent aux applications fort couplebasse vitesse (traction, robotique en prise directe) ou forte puissance-grande vitesse [2].

La structure polyentrefer leur permet d'atteindre des performances couple et puissance massiques très intéressantes à condition de leur adjoindre un convertisseur d'alimentation à fréquences élevées (jusqu'à quelques kHz).

En général, il est à prévoir un fort surdimensionnement des convertisseurs pour l'alimentation de machines à réluctance variable. On emploie souvent l'expression de «mauvais facteur de puissance » pour le qualifier. Pour pallier à ce grave inconvénient, surtout dans le cas des machines de forte puissance, nous avons mené des travaux d'optimisation des formes du courant d'alimentation au L.E.SI.R. (E.N.S. de Cachan) conjointement avec le Laboratoire d'Electrotechnique des Universités Paris VI et XI où sont conçus et étudiés des prototypes de machines à réluctance variable polydiscoïdes. Une première recherche de formes optimales du courant d'alimentation a été effectuée sur des critères de maximisation des rapports couple moyen sur courant efficace (pertes Joule) et couple moyen sur courant maximal [1]. Les formes obtenues, si elles permettaient une optimisation de la machine, ne conduisaient malheureusement pas à un dimensionnement minimal du convertisseur. C'est donc une nouvelle optimisation que nous allons effectuer en considérant l'aspect dimensionnement des convertisseurs alimentant soit des machines monophasées, soit des machines polyphasées par éléments (multimonophasées ou multistack). 
Ces dernières ne possèdent pas de couplage magnétique entre phases, leur étude peut donc être déduite de celle des machines monophasées, le couple résultant étant calculé par sommation des couples instantanés produit par chaque élément.

\section{Modèle de la machine. Alimentations et facteurs} de dimensionnement.

2.1 PRINCIPE ET MODÈLE DE LA MACHINE A RÉLUCTANCE VARIABLE. - Dans cette étude générale, nous ne prenons pas en compte les pertes Joule (elles sont, en général, faibles dans ces machines d'assez forte puissance). D'autre part, on s'intéresse à la maximisation de la puissance électrique convertie qui comprend la puissance mécanique correspondant au couple électromagnétique mais aussi les pertes fer. Si les matériaux magnétiques sont convenablement choisis en fonction des fréquences utilisées, celles-ci peuvent être faibles. Nous pouvons ainsi représenter le fonctionnement complet de la machine par la seule fonction perméance de l'espace (formes des motifs magnétiques, dents ou plots) et des ampères-tours (prise en compte de la saturation). La perméance est notée $z(\theta, j)$, elle est définie comme le rapport du flux aux ampères-tours. $\theta$ est l'angle électrique et :

$$
\theta=p \cdot \theta_{\mathrm{m}}
$$

où $p$ est le nombre de motifs magnétiques identiques du rotor et $\theta_{\mathrm{m}}$ est l'angle mécanique. Les ampèrestours sont notés $j$ et valent :

$$
j=n \cdot i
$$

où $i$ est le courant d'alimentation et $n$ le nombre de spires du bobinage.

La figure 2.1 représente une structure élémentaire monophasée à entrefer axial. Sur une période électrique, deux positions du rotor sont remarquables:

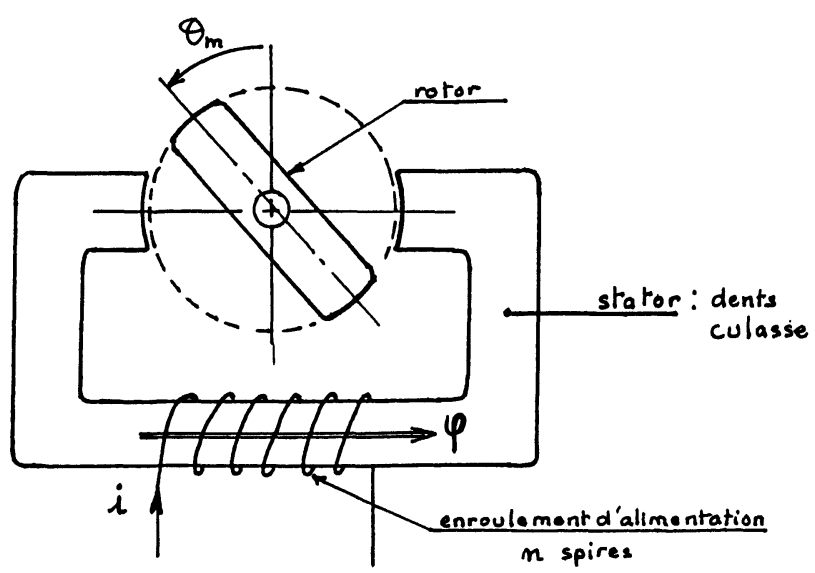

Fig. 2.1. - Structure élémentaire d'une machine à réluctance variable.

[Elementary structure of a reluctance machine (R.M.).] celle d'opposition où la perméance est minimale et celle de conjonction où elle est maximale.

Une machine à réluctance variable quelconque peut ainsi être représentée électromagnétiquement par le réseau de courbes $\varphi(j)$ paramétré en $\theta$ (Fig. 2.2) où $\varphi$ est le flux par spire. On montre alors que le couple instantané a pour expression [3] :

$$
c=\frac{\partial W_{\mathrm{em}}^{\prime}(j, \theta)}{\partial \theta}
$$

en définissant la coénergie $W_{\mathrm{em}}^{\prime} \operatorname{par} \int_{0}^{j} \varphi \cdot \mathrm{d} j^{\prime}$ (voir Fig. 2.2).

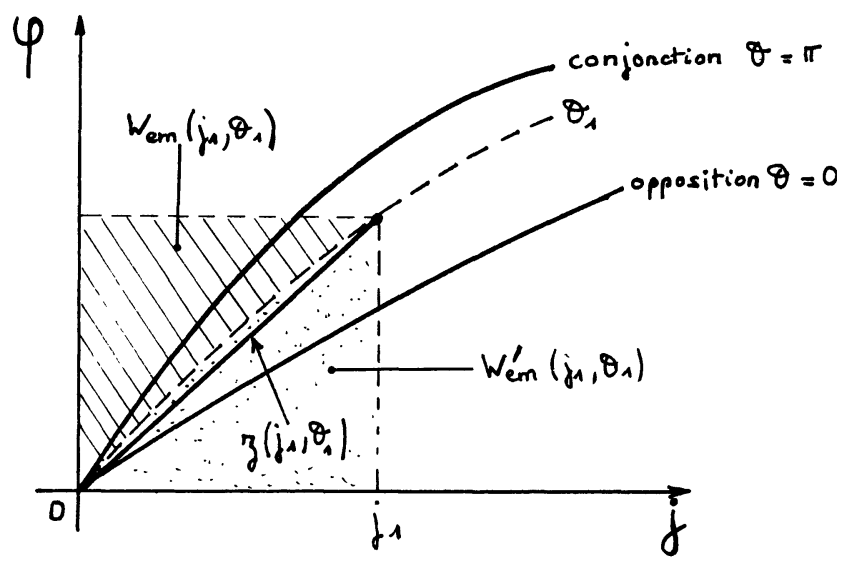

Fig. 2.2. - Réseau de caractéristiques représentant électromagnétiquement la machine.

[Electromagnetic model of the machine.]

L'énergie $w$ convertie par cycle est alors égale à l'aire du cycle parcouru dans le plan $\varphi(j)$ :

$$
w=\oint z(j, \theta) \cdot j \cdot \mathrm{d} j .
$$

Le couple moyen $\langle c\rangle$ est donné par l'expression :

$$
\langle c\rangle=p w / 2 \pi
$$

car la conservation de l'énergie est représentée par l'égalité :

$$
\langle c\rangle \cdot \Omega=f \cdot w .
$$

En régime non saturé, la relation entre flux et ampères-tours est linéaire :

$$
\varphi=z(\theta) \cdot j .
$$

Le couple instantané $c$ s'exprime alors simplement par les deux expressions suivantes équivalentes :

$$
c=\frac{p}{2} j^{2} \frac{\mathrm{d} z}{\mathrm{~d} \theta}
$$

ou

$$
c=-\frac{p}{2} \varphi^{2} \frac{\mathrm{d} \mathcal{R}}{\mathrm{d} \theta}
$$


où $\mathcal{R}=z^{-1}$ est la réluctance. L'expression (2.8) est applicable directement lorsque la machine est alimentée en courant alors que l'expression (2.9) simplifie le calcul dans le cas d'une alimentation à flux forcé ou en tension.

On remarque que le signe du courant ou du flux est sans influence sur le signe du couple mais que ce dernier ne dépend que du signe de la variation de la perméance par rapport à $\theta$. Pour avoir un fonctionnement moteur (ou génératrice), il suffira « d'injecter plus de courant " lorsque la perméance est croissante (ou décroissante) que lorsqu'elle est décroissante (ou croissante). Nous nous restreindrons ici à l'étude du fonctionnement moteur sachant que le fonctionnement génératrice en découle (car la fonction $z(\theta)$ est paire par symétrie des motifs magnétiques).

A partir de ces équations en régime linéaire, nous allons rappeler quels sont les principaux modes d'alimentations possibles et la terminologie correspondante.

\subsection{MODES D'ALIMENTATION.}

2.2.1 Machine non excitée. - La machine ne comporte qu'un seul bobinage par phase et il produit tous les ampères-tours. Le signe du couple étant indépendant du signe de ces derniers, deux types d'alimentation sont possibles pour le même couple instantané produit. En effet, supposons que l'on alimente la machine en lui imposant périodiquement un courant positif (en traits pleins sur Fig. 2.3). On peut obtenir un couple moyen non nul. Le courant est unidirectionnel et sa période est identique à celle de l'onde de perméance.

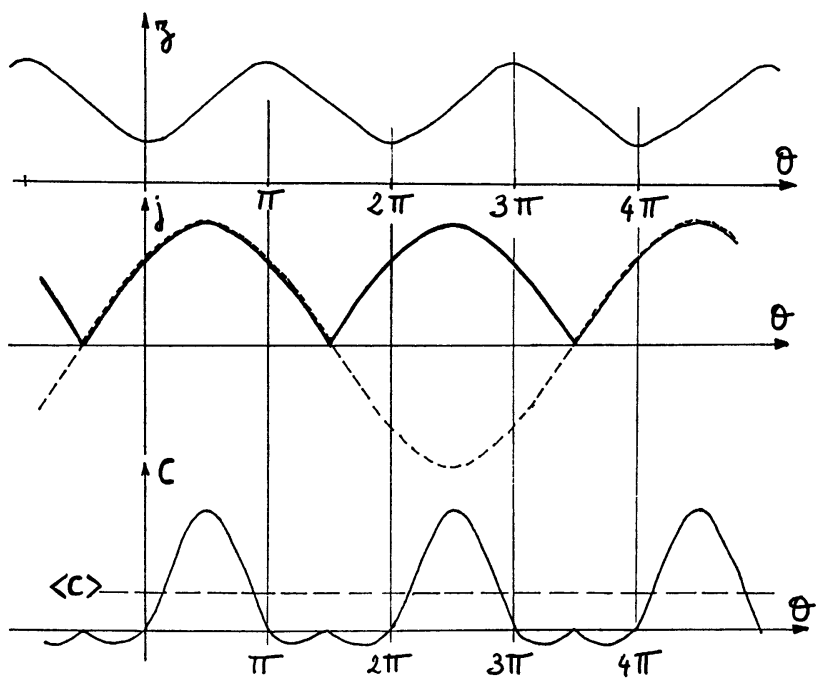

Fig. 2.3. - Alimentation en régime non excité en courant unidirectionnel ou bidirectionnel.

[Unidirectional or bidirectional current supply in unexcited mode.]
Maintenant, changeons le signe de ce courant initial une période sur deux (en trait interrompu sur Fig. 2.3). Le couple instantané n'est pas modifié, mais cette fois le courant est bidirectionnel à valeur moyenne nulle et sa période correspond à deux périodes de la fonction perméance. Ces deux types d'alimentation sont utilisés [4, 5].

2.2.2 Machine excitée. - Ici, sur chaque phase de la machine sont utilisés deux bobinages distincts supposés parfaitement couplés. Les ampères-tours produits par chacun d'eux s'ajoutent algébriquement. L'un d'eux, dit bobinage d'excitation, produit des ampères-tours constants. L'autre, dit bobinage d'alimentation, produit des ampères-tours variables à la fréquence de variation de la perméance. Ces derniers peuvent être soit bidirectionnels à valeur moyenne nulle (mode d'excitation 1). Ils permettent une excursion autour de ceux d'excitation. Ils peuvent aussi être unidirectionnels (mode 2), ils ne font alors qu'accroître la valeur des ampères-tours d'excitation (Fig. 2.4).

Lès ampères-tours continus sont en général produits à moindre coût. C'est là l'intérêt majeur de la machine excitée.

2.2.3 Calcul du couple moyen suivant les modes d'alimentation. - Nous allons montrer qu'il existe deux fréquences du courant d'alimentation qui permettent d'obtenir un couple moyen non nul. Ceci constitue une explication théorique simplifiée des modes d'alimentation précédemment décrits.

Les hypothèses de calculs sont le fonctionnement en régime linéaire et la limitation aux fondamentaux

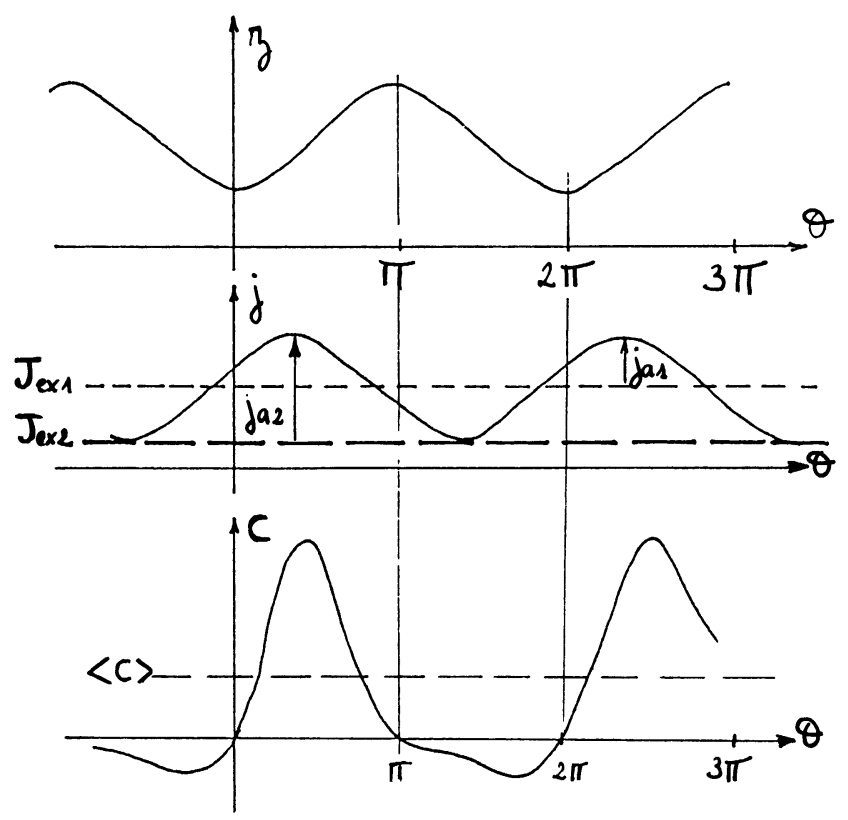

Fig. 2.4. - Alimentation en régime excité (modes 1 ou 2).

[Supply in excited mode ( 1 or 2$)$.] 
des ampères-tours et de l'onde spatiale de perméance.

Soient les ampères-tours $j$ et l'onde de perméance $z$ :

$$
\begin{aligned}
c=\frac{p}{2} Z_{1}\left[J_{0}^{2} \sin \theta+J_{0} J_{\mathrm{aM}} \cos ((k-1) \theta+\beta)-J_{0} J_{\mathrm{aM}} \cos ((k+1) \theta+\beta)+\right. \\
\left.\quad+\frac{J_{\mathrm{aM}}^{2}}{2} \sin \theta+\frac{J_{\mathrm{aM}}^{2}}{4} \sin ((2 k-1) \theta+2 \beta)-\frac{J_{\mathrm{aM}}^{2}}{4} \sin ((2 k+1) \theta+2 \beta)\right]
\end{aligned}
$$

Le couple moyen est non nul dans deux cas :

* $k=+1$ (ampères-tours à la même fréquence que l'onde de perméance)

$$
\langle c\rangle=(p / 2) Z_{1} J_{0} J_{\mathrm{aM}} \cos \beta .
$$

$\mathrm{Ce}$ cas correspond soit au régime excité dans lequel $J_{0}$ représente l'excitation du mode 1 (Fig. 2.4), soit au régime non excité en courant unidirectionnel, $J_{0}$ représente alors simplement la valeur moyenne des ampères-tours. Dans ces conditions, la machine ne peut donc fournir un couple que si la valeur moyenne des ampères-tours est non nulle. Le couple moyen est maximal pour $\beta=\pi / 2$. Ce couple est dit « couple hybride» dans les machines excitées par courants ou par aimants [6].

* $k=1 / 2$ (ampères-tours ayant une période double de celle de la perméance)

$$
\langle c\rangle=(P / 8) Z_{1} J_{\mathrm{aM}}^{2} \cdot \sin 2 \beta .
$$

Ceci correspond au régime non excité avec alimentation en courant bidirectionnel. Le couple moyen est indépendant de $J_{0}$ que l'on a intérêt à annuler pour minimiser le couple pulsatoire ainsi que les pertes Joule.

Ce couple proportionnel au carré du courant est dit « couple de réluctance pure».

On remarque que, dans les deux cas, le couple est proportionnel à la variation absolue de la perméance. Une étude générale prenant en compte les harmoniques éventuels des ampères-tours et de l'onde de perméance a montré la complexité des interactions et l'intérêt des harmoniques de courant même lorsque l'onde spatiale de perméance est sinusoïdale [7].

2.3 DÉFINITIONS DES FACTEURS DE DIMENSIONNEMENT. - Pour obtenir la variation de leur caractéristique mécanique externe $c(\Omega)$, il est nécessaire d'alimenter les machines avec des convertisseurs statiques. Aussi est-il important de se soucier de leur dimensionnement, celui-ci influant sur le coût, le volume, la masse et même le rendement de l'ensemble.

2.3.1 Définition en régime non excité. - En fonction de sa charge, la machine demande à son convertis- seur une certaine puissance électrique transmise par des ondes de tension et de courant non nécessairement continues ou sinusoïdales. Leurs valeurs maximales et efficaces sont des contraintes pour les composants semi-conducteurs. On peut parler de puissance de dimensionnement du convertisseur en volts-ampères (V.A) ou "puissance silicium », celleci est toujours supérieure ou égale à la puissance active convertie électriquement. L'idéal correspond à l'égalité.

En électrotechnique, on utilise classiquement le facteur de dimensionnement $\delta$ défini par:

$$
\delta=S / P
$$

où $P$ et $S$ sont respectivement les puissances active et apparente. Il vaut $1 / \cos \varphi$ en courant et tension sinusoïdaux, $\varphi$ est alors le déphasage entre eux. (On parle aussi de facteur de puissance $f_{\mathrm{p}}=1 / \delta$ donc $f_{\mathrm{p}} \leqslant 1$.)

Les convertisseurs de puissance travaillent le plus souvent en courant et tension non sinusoïdaux. Par conséquent le précédent facteur de dimensionnement ne peut être valablement utilisé. Il a donc fallu introduire deux nouveaux rapports définis par analogie avec $\delta[8]$ : ce sont les coefficients $\delta^{\prime}$ et $\delta^{\prime \prime}$ définis par :

$$
\begin{aligned}
& \delta^{\prime}=U_{\mathrm{M}} \cdot I_{\text {eff }} / P \\
& \delta^{\prime \prime}=U_{\mathrm{M}} \cdot I_{\mathrm{M}} / P
\end{aligned}
$$

où $U_{\mathrm{M}}$ est la tension maximale aux bornes d'une phase de la machine et $I_{\text {eff }}, I_{\mathrm{M}}$ sont respectivement les courants efficace et maximal traversant cette phase. Ces rapports sont applicables à tout type d'alimentation. En particulier, en régime sinusoïdal, ces coefficients s'expriment simplement en fonction $\mathrm{du} \ll \cos \varphi »$ par :

$$
\delta^{\prime}=\sqrt{2} / \cos \varphi \quad \text { et } \quad \delta^{\prime \prime}=2 / \cos \varphi .
$$

Tous les semi-conducteurs doivent être dimensionnés pour la tension maximale qu'ils supportent. En revanche, le choix du calibre courant dépend du semi-conducteur et de son utilisation. Aussi, le facteur de dimensionnement $\delta^{\prime}$ s'utilisera-t-il pour dimensionner les convertisseurs à thyristors et/ou à diodes fonctionnant en commutation assistée car ces 
composants ne possèdent pas d'effet de saturation et ont de plus une inertie thermique suffisante. Le facteur $\delta^{\prime \prime}$ s'appliquera au dimensionnement des convertisseurs à commutation forcée où le « pouvoir de coupure » est limité soit par le composant (transistor, thyristor G.T.O.) soit par les circuits auxiliaires de blocage.

On remarquera que ces composants ne prennent pas en compte les structures de convertisseurs. Ils sont tout à fait généraux et se calculent à partir des équations de la machine alimentée en tension ou en courant. Pour la comparaison de différentes structures et en vue d'effectuer un choix, nous introduisons la notion de facteur de dimensionnement global $\delta_{\mathrm{g}}^{(i)}$.

Nous prenons alors en compte le nombre d'interrupteurs commandés qui dépend de la structure de convertisseur (le passage d'un courant bidirectionnel peut nécessiter deux fois plus d'interrupteurs qu'un courant unidirectionnel) et du couplage électrique (étoile ou polygonal) éventuel des phases de la machine. Toutefois, pour effectuer l'optimisation générale, nous n'étudierons que les facteurs de dimensionnement élémentaires $\delta^{\prime}$ et $\delta^{\prime \prime}$. Nous ne présenterons, essentiellement, que les résultats concernant $\delta^{\prime \prime}$, prenant en compte le fait que la quasi-totalité des machines à réluctance variable doit fonctionner en commutation forcée pour une utilisation au maximum de leurs possibilités. Nous montrerons sur un exemple comment s'effectue le calcul des $\delta_{\mathrm{g}}^{(i)}$ (Annexe 1).

2.3.2 Définitions en régime excité. - Le régime excité défini au $\$ 2.2 .2$ nécessite la création d'ampères-tours continus soit par des aimants permanents soit par un bobinage d'excitation. Dans ce dernier cas, il faut lui fournir ses pertes Joule. Cette excitation peut donc avoir des coûts d'exploitation et d'équipement inférieurs à ceux du ou des bobinages d'alimentation. Nous allons prendre en compte ce coût dans l'expression des facteurs de dimensionnement en régime excité, nous utiliserons un coefficient $\alpha$ pondérateur du coût des ampères-tours d'excitation par rapport à ceux d'alimentation [8].

Soient $J_{\text {eff eq }}$ et $J_{M \text { eq }}$ les ampères-tours efficaces et maximaux équivalents définis par :

$$
\begin{aligned}
& J_{\text {eff eq }}^{2}=J_{\text {a eff }}^{2}+\alpha J_{\text {ex }}^{2} \\
& J_{\mathrm{M} \mathrm{eq}}=J_{\mathrm{aM}}+\alpha J_{\mathrm{ex}}
\end{aligned}
$$

où $J_{\mathrm{a}}$ et $J_{\mathrm{ex}}$ sont les ampères-tours d'alimentation et d'excitation en modes 1 ou 2 (voir $\$ 2.2 .2$ ).

Le coefficient $\alpha$ sera théoriquement compris entre 0 et 1 selon la complexité relative des convertisseurs d'excitation et d'alimentation. Dans un cas général, il semble que 0,2 soit une valeur réaliste [7]. L'excitation n'est intéressante que si $\alpha$ est inférieur à $0,5[8]$.

On définit alors les facteurs de dimensionnement en régime excité comme suit :

$$
\begin{aligned}
& \delta^{\prime}=\frac{U_{\mathrm{M}} \cdot \frac{J_{\text {eff eq }}}{n}}{P} \\
& \delta^{\prime \prime}=\frac{U_{\mathrm{M}} \cdot \frac{J_{\mathrm{M} \mathrm{eq}}}{n}}{P}
\end{aligned}
$$

où $n$ est le nombre de spires de l'enroulement d'alimentation.

On affecte les $\delta^{(i)}$ de l'indice 1 ou 2 si le mode d'alimentation en régime excité est 1 ou 2 . On fait précéder cet indice de l'indice 0 lorsque l'on considère le dimensionnement du convertisseur d'alimentation seul (sans prise en compte de l'excitation).

$\mathrm{Si}$ on considère l'exemple de la figure 2.4 en mode 2 , les ampères-tours efficaces et maximaux d'alimentation sont plus élevés qu'en mode 1 , en revanche $J_{\text {ex2 }}$ est inférieur à $J_{\text {ex1 }}$. Dans le cas où ces ampères-tours sont sinusoïdaux, on $\mathrm{a}$ :

$$
\delta_{02}^{\prime}=\sqrt{3} \delta_{01}^{\prime} \quad \text { et } \quad \delta_{02}^{\prime \prime}=2 \cdot \delta_{01}^{\prime \prime}
$$

donc

$$
\frac{\delta_{02}^{\prime}}{\delta_{01}^{\prime}}<\frac{\delta_{02}^{\prime \prime}}{\delta_{01}^{\prime \prime}}
$$

Donc, pour le même état magnétique de la machine et donc le même couple, le facteur de dimensionnement dépend du mode d'alimentation. C'est ici qu'il faut alors prendre en compte la notion de facteur de dimensionnement global. En mode 2, le courant d'alimentation est unidirectionnel alors qu'en mode 1 , il est bidirectionnel. Si le nombre d'interrupteurs doit être doublé, on obtient :

$$
\delta_{\mathrm{g} 02}^{\prime}=\frac{\sqrt{3}}{2} \delta_{\mathrm{g} 01}^{\prime} \quad \text { et } \quad \delta_{\mathrm{g} 02}^{\prime \prime}=\delta_{\mathrm{g} 01}^{\prime \prime}
$$

donc

$$
\frac{\delta_{\mathrm{g} 02}^{\prime}}{\delta_{\mathrm{g} 01}^{\prime}}>\frac{\delta_{\mathrm{g} 02}^{\prime \prime}}{\delta_{\mathrm{g} 01}^{\prime \prime}}
$$

2.4 INTÉRÊT D'UNE OPTIMISATION DE LA FORME DU COURANT. - Nous avons évoqué dans l'introduction les mauvais facteurs de dimensionnement habituellement rencontrés dans les ensembles convertisseursmachines à réluctance variable. Le facteur $\delta$ " atteint couramment des valeurs de l'ordre de 40 [4], les plus faibles valeurs rencontrées dans la littérature étant peu inférieures à 10 [9].

A titre comparatif, pour une machine classique (synchrone ou asynchrone) alimentée en courant alternatif, alimentée en tension et courant sinusoïdaux (donc bidirectionnels) on a $\delta^{\prime \prime}=\frac{2}{\cos \varphi}$ soit nécessairement : $\delta^{\prime \prime} \geqslant 2$. Pour une machine à courant continu : $\delta^{\prime \prime}=1$.

Si la réversibilité du système doit être considérée, il faut raisonner avec le facteur de dimensionnement global. L'étude doit alors prendre en compte d'autres 
paramètres comme la nature de la source d'alimentation.

Pour des raisons évidentes de diminution du coût, du volume et de la masse et une augmentation du rendement, il a semblé intéressant de rechercher les formes du courant d'alimentation permettant de minimiser les facteurs de dimensionnement $\delta^{\prime}$ et $\delta^{\prime \prime}$.

Nous allons travailler en considérant les caractéristiques du circuit magnétique de la machine, c'est-àdire le flux par spire et les ampères-tours liés entre eux par la perméance avec la relation :

$$
\varphi=z(\theta, j) \cdot j(\theta) .
$$

Il sera, ainsi, toujours possible d'ajuster le nombre de spires de l'enroulement d'alimentation d'une phase de la machine pour se trouver dans la plage courant-tension optimale des composants disponibles :

$$
u \cdot i=n \frac{\mathrm{d} \varphi}{\mathrm{d} t} \cdot \frac{j}{n}=\frac{\mathrm{d} \varphi}{\mathrm{d} t} \cdot j=\omega\left[\frac{\mathrm{d} \varphi}{\mathrm{d} \theta} \cdot j\right] .
$$

Il apparaît dès lors que les facteurs de dimensionnement, calculés à partir du produit $u . i$ (tensioncourant), ne dépendent pas du nombre de spires de la machine mais uniquement des caractéristiques de son circuit magnétique. Ils peuvent être ainsi déterminés uniquement en connaissant la fonction perméance $z(\theta, j)$.

D'après les expressions (2.4), (2.5), (2.6) et (2.21) et si l'on considère l'égalité des puissances mécanique et électrique, on montre que :

$$
\delta^{(i)}=\frac{\left\{\frac{\mathrm{d}(z(\theta, j) \cdot j)}{\mathrm{d} \theta}\right\} \cdot\{j\}}{\frac{1}{2 \pi} \oint z(\theta, j) \cdot j \cdot \mathrm{d} j} .
$$

Les expressions entre accolades représentent soit la valeur efficace, soit la valeur maximale. La vitesse de rotation (ou la fréquence d'alimentation) n'intervient plus. Seul le couple demandé peut avoir une influence sur $\delta^{(i)}$ si l'on considère la saturation. En effet, imaginons une alimentation imposant une certaine onde de courant de forme figée et dont on fait varier l'amplitude pour changer la valeur du couple. En régime linéaire, l'onde de tension induite a toujours la même forme (fonction $z$ indépendante de $j$ ) mais en régime de saturation, son allure évolue en fonction du niveau d'excitation magnétique, les facteurs de dimensionnement sont alors modifiés. Ceci est une différence très importante des machines à réluctance variable par rapport aux machines classiques, dans lesquelles la puissance de dimensionnement du convertisseur reste sensiblement proportionnelle à la puissance électrique demandée.

2.5 MODÈle SIMPLIFIÉ DE LA MACHINE. NORMALISATION. - La machine étant complètement définie par son réseau de courbes $\varphi(j, \theta)$ (Fig. 2.2), nous avons choisi le « modèle à 3 pentes » (Fig. 2.5), déjà éprouvé $[8,10]$ dans lequel $z_{0}, z_{\mathrm{c}}$ et $z_{\mathrm{s}}$ représentent les pentes des trois portions de droite. $z_{0}$ et $z_{\mathrm{c}}$ sont les perméances à l'opposition et à la conjonction en régime linéaire.

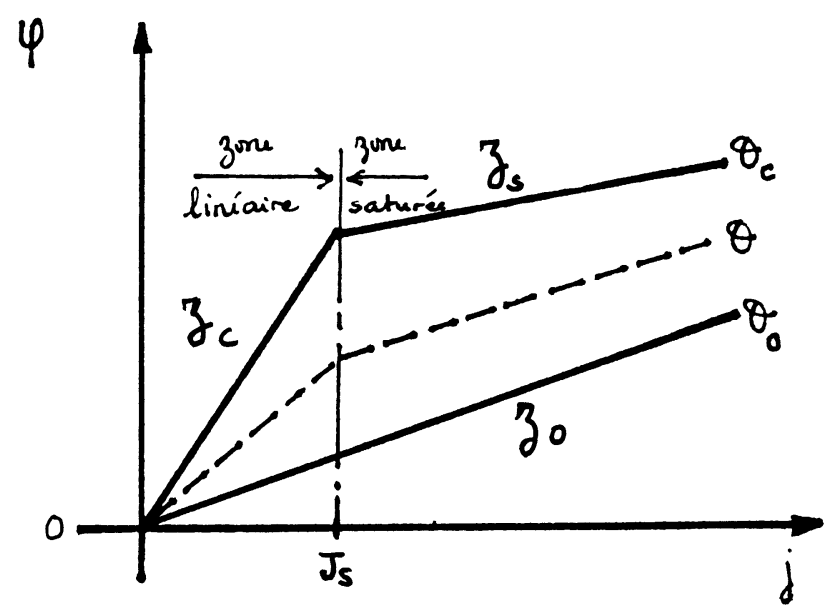

Fig. 2.5. - Modèle électromagnétique à trois pentes.

[Three-slope electromagnetic model.]

La perméance $z$ (rapport $\varphi / j$ ) est normalisée par rapport à $\left(z_{\mathrm{c}}+z_{0}\right) / 2$.

$$
Z=\frac{z}{\frac{z_{\mathrm{c}}+z_{0}}{2}} .
$$

On notera $D Z N$ la variation relative de la perméance en régime linéaire :

$$
D Z N=\frac{z_{\mathrm{c}}-z_{0}}{z_{\mathrm{c}}+z_{0}}
$$

$(D Z N \rightarrow 0$ pour un «cycle fermé », $D Z N \rightarrow 1$ pour un « cycle ouvert ».)

La valeur de $D Z N$ tend à diminuer lorsque, sur une structure donnée, on augmente le nombre de motifs.

Le paramètre $K$ représente la saturation, la figure 2.6 donne les allures types du modèle à 3 pentes en fonction du signe de $K$.

$$
K=\frac{z_{\mathrm{s}}-z_{0}}{z_{\mathrm{c}}-z_{0}} .
$$

Les trois cas présentés figure 2.6 représentent, en fait, des régimes de fonctionnement différents des machines. Lorsque $K$ est négatif, la machine est fortement saturée et on passe assez brutalement de la zone linéaire à la zone saturée. Lorsque $K$ est positif, la machine est peu saturée. On représente alors le coude de saturation dans lequel on travaille mais le domaine de validité du modèle est alors limité. 

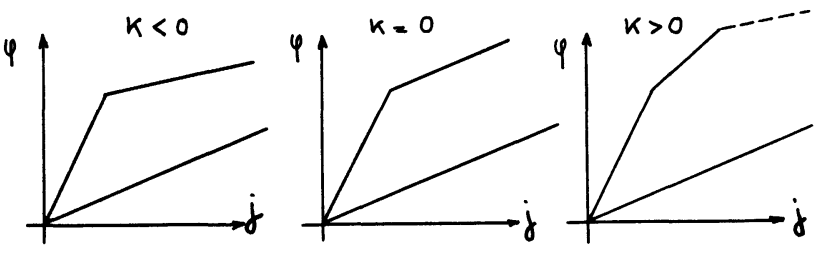

Fig. 2.6. - Différentes allures de la caractéristique magnétique en fonction de $K$.

[Different shapes of magnetic characteristics as a function of $K$.]

L'allure de l'onde spatiale de perméance $z(\theta)$, à ampères-tours constants, peut être choisie sinusoïdale ou telle que la variation de réluctance soit sinusoïdale, trapézoïdale ou décomposée en série de Fourier. On suppose que cette fonction est indépendante du niveau d'excitation du fer. Dans tous les cas, elle présente une symétrie par rapport à $\pi$ (symétrie des motifs plots ou dents).

Les ampères-tours normalisés (notés $J$ ) le sont par rapport. aux ampères-tours de saturation $J_{\mathrm{s}}$ (Fig. 2.5) :

$$
J=j / J_{\mathrm{s}} .
$$

Ces deux normalisations de base imposent celles du flux et du couple, on notera $F L U X$ le flux et $C$ le couple normalisés :

$$
\begin{gathered}
F L U X=\frac{\varphi}{\frac{z_{\mathrm{c}}+z_{0}}{2} \cdot J_{\mathrm{s}}} \\
C=\frac{c}{p \cdot \frac{z_{\mathrm{c}}+z_{0}}{2} \cdot J_{\mathrm{s}}} .
\end{gathered}
$$

On notera $U / \omega$ la tension normalisée par spire et par radian par seconde :

$$
\frac{U}{\omega}=\frac{u}{n \cdot \omega \cdot \frac{z_{\mathrm{c}}+z_{0}}{2} \cdot J_{\mathrm{s}}} .
$$

Lorsqu'il s'agira de la valeur maximale, on écrira $U_{\mathrm{M}} / \omega$.

A partir d'un tel modèle et avec les normalisations choisies, le couple normalisé a pour valeur l'aire du cycle divisée par $2 \pi$ (Fig. 2.9).

On montre alors simplement qu'en régime linéaire, si on remplit totalement le cycle :

$$
C_{\mathrm{L}}=\frac{D Z N}{2 \pi}
$$

et dans le modèle avec $K<0$, si $J_{x}$ représente les ampères-tours normalisés de l'intersection des courbes à l'opposition et à la conjonction pour un cycle totalement « rempli », on obtient le couple normalisé maximal :

$$
\begin{aligned}
C_{\mathrm{M}} & =\frac{D Z N}{2 \pi} \cdot J_{x} \\
J_{x} & =1-\frac{1}{K} .
\end{aligned}
$$

Ce remplissage du cycle ne peut être obtenu qu'en injectant un créneau de courant pendant la croissance de la fonction perméance (en moteur).

\section{L'optimisation et ses résultats essentiels.}

3.1 L'Alimentation @PTIMALE. - Nous avons écrit un programme spécifique d'optimisation de la forme du courant en discrétisant toutes les variables sur une période d'alimentation [7].

Les ampères-tours sont alors représentés par un nombre $N$ d'échantillons qui constituent les $N$ variables inconnues de l'optimisation. Les données du problème sont la forme de l'onde spatiale de perméance $z(\theta)$, les paramètres $D Z N$ et $K$. De plus, au-delà de la zone linéaire, il est indispensable d'imposer une contrainte sur la valeur du couple car les formes optimales du courant ne peuvent être homothétiques lorsque le couple augmente et les facteurs de dimensionnement dépendent ainsi du couple moyen (voir fin du $\$ 2.4$ ).

Après avoir mené une étude quasi systématique des cas possibles (valeurs de $D Z N, K, \alpha$ en régime excité et forme d'onde spatiale de perméance $z(\varphi)$ variées) les formes optimales d'onde de courant qui minimisent $\delta^{\prime}$ ou $\delta^{\prime \prime}$ se sont révélées telles que les tensions induites correspondantes étaient en créneaux symétriques bidirectionnels à 2 ou 3 paliers (Fig. 3.1a pour le régime non excité et Fig. 3.1b pour le régime excité).

En régime permanent, le flux est périodique, les deux paliers non nuls de tension ont une amplitude égale et par conséquent sont de durée identique. En régime excité ou non, quels que soient le niveau de saturation atteint (ou le couple demandé) et les caractéristiques de la machine, l'alimentation optimale peut être définie par seulement trois paramètres qui sont : le rapport de la tension maximale à la pulsation $U_{\mathrm{M}} / \omega$ qui représente le flux maximal, l'angle d'avance à l'opposition $\psi$ que l'on peut appeler angle interne et, pour le régime non excité l'angle mort $\theta_{0}$, pour le régime excité, le flux moyen $\varphi_{0}$ qui définit les ampères-tours d'excitation en mode 1 ou 2 selon le cas choisi.

Le programme réalisé nous permet d'optimiser dans les 3 types d'alimentation prévus et éventuellement d'optimiser à ampères-tours d'excitation imposés. Il s'avère donc que l'alimentation en tension en créneaux et en ampères-tours unidirectionnels à la fréquence de la fonction perméance (couple hybride) est optimale dans les machines à réluctance variable à phases non couplées magnétiquement.

Nous avons, à partir de ces résultats, mis au point un nouveau programme d'optimisation mettant en 

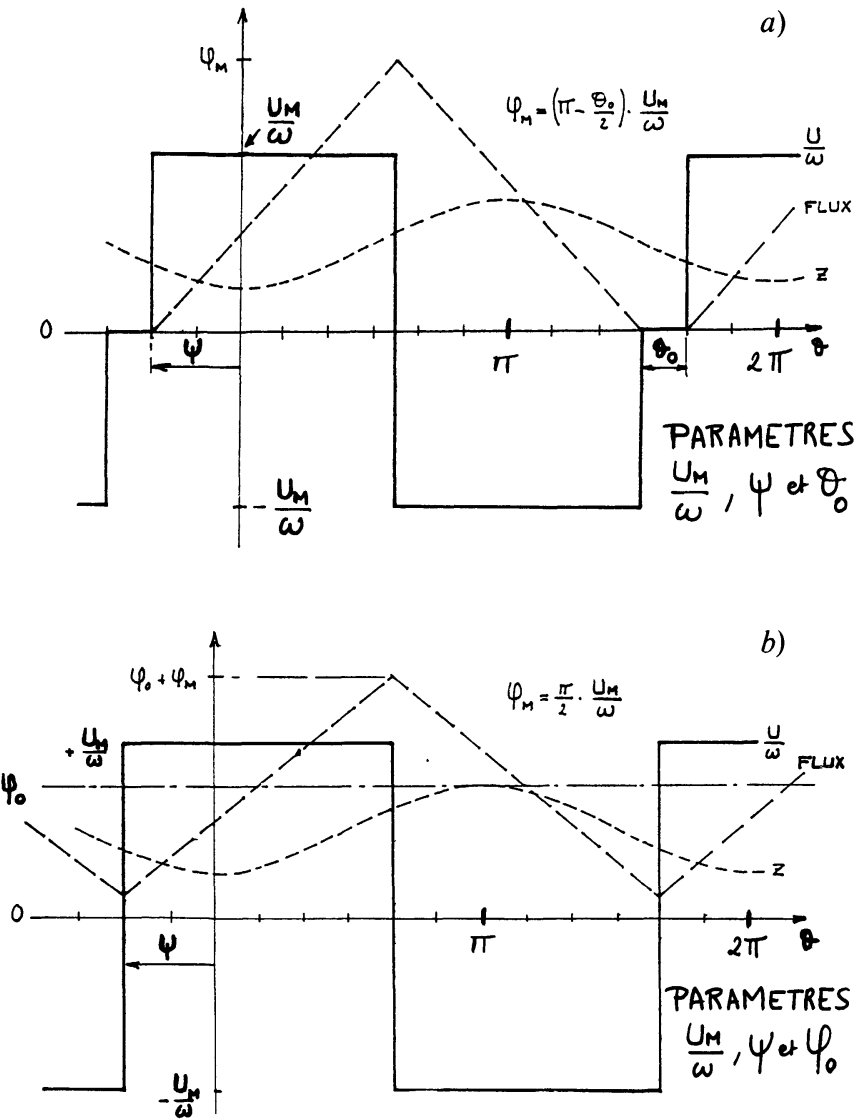

Fig. 3.1. - Alimentation optimale, 3.1a : en régime non excité, $3.1 \mathrm{~b}$ : en régime excité.

[Optimal supply, 3.1a : unexcited mode, 3.2b : excited mode.]

œuvre la méthode du simplexe mieux adaptée à un faible nombre d'inconnues. Celui-ci nous a permis de réaliser une étude paramétrique en vue de rechercher la machine idéale pour le convertisseur, c'est-à-dire minimisant la taille de ce dernier sans augmenter pour autant celle de la machine, les paramètres étant $D Z N, K$ et l'onde spatiale de perméance $z(\theta)$. Parallèlement à cette optimisation, nous avons étudié l'influence des caractéristiques de l'alimentation sur le couple, c'est-à-dire sur son contenu harmonique et sur un nouveau critère qui a semblé important dans les machines multimonophasées de forte puissance : l'ondulation crête à crête du couple rapportée au couple moyen et notée $\Delta C /\langle C\rangle$. En effet, le couple généralement très pulsatoire est responsable de contraintes mécaniques importantes.

Rappelons que dans une machine polyphasée à $q$ phases, il se produit un «filtrage » des harmoniques $\mathrm{du}$ couple produit par une phase, il ne reste en effet que les rangs multiples de $q$. Ainsi, si le couple instantané ne comprend pas d'harmoniques de rang multiple de 3 , le couple d'une machine triphasée est parfaitement constant.
Certains résultats seront appliqués au cas particulier du prototype polydiscoïde de $200 \mathrm{~kW}$ à 500 tours/min $(200 \mathrm{~Hz})$ du Laboratoire d'Electrotechnique d'Orsay dont les caractéristiques normalisées sont les suivantes :

- onde spatiale de perméance sinusoïdale,

$-D Z N=0,5$,

$-K=0,2$,

- couple nominal normalisé $\langle C\rangle=0,11$.

3.2 RÉSUlTATS CONCERNANT LES FACTEURS DE DIMENSIONNEMENT. - En régime linéaire comme en saturation, l'alimentation en tension en créneaux offre une importante amélioration par rapport aux diverses alimentations en courant en ce qui concerne le dimensionnement du convertisseur. Cette amélioration s'observe quelles que soient les caractéristiques de la machine, cependant, elle est d'autant plus significative que la variation de perméance $D Z N$ est importante. Un tel exemple est donné à la figure 3.2 où une minimisation de $\delta^{\prime \prime}$ est effectuée sur la même machine (perméance sinusoïdale, linéaire, non excitée) mais l'une est alimentée par un courant en arches de sinusoïde (alimentation de type à résonance [11]) et l'autre par des créneaux de tension.

Lorsqu'on effectue les optimisations en tenant compte de la saturation, les calculs montrent (selon

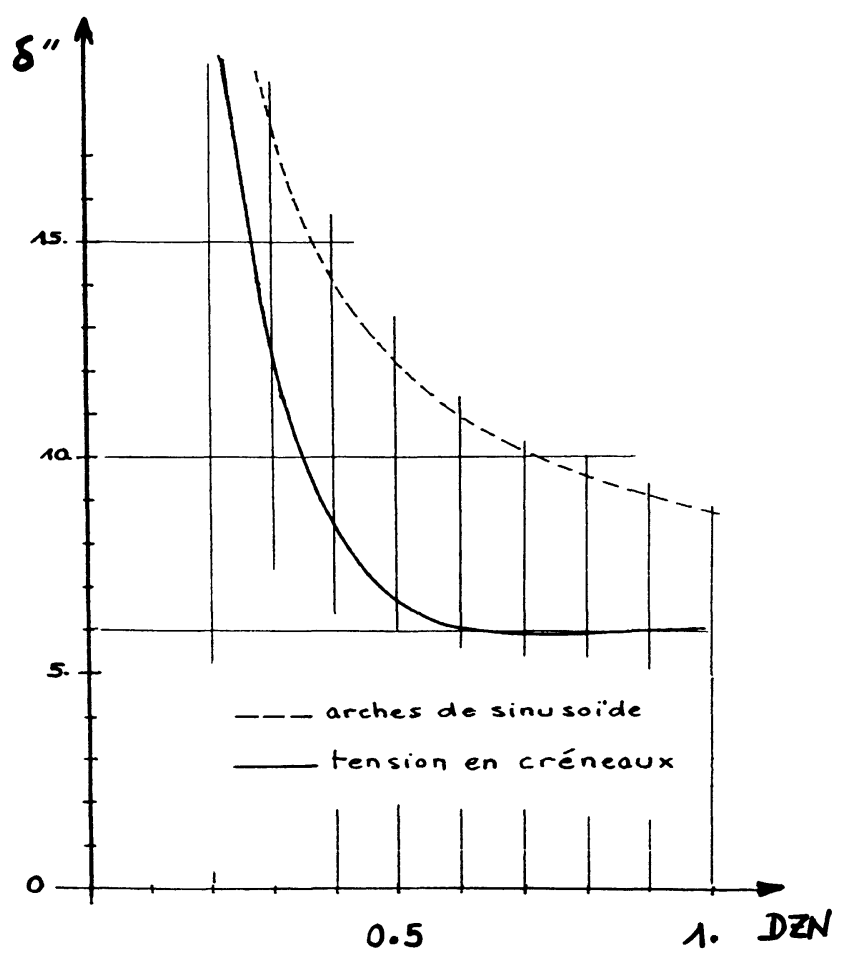

Fig. 3.2. - Influence de la variation relative de perméance sur le facteur de dimensionnement. Avantage de l'alimentation en tension en créneaux.

[Influence of the variation of permeance on the size power ratio (S.P.R.). Advantage of the square-wave voltage supply.] 
les valeurs du paramètre $K$ qui caractérise la saturation) une amélioration des facteurs de dimensionnement à l'entrée en saturation puis une dégradation de ceux-ci lorsque l'on approche de la limite du couple maximal convertible électromagnétiquement (Expression (2.31)) par la machine (Fig. 3.3).

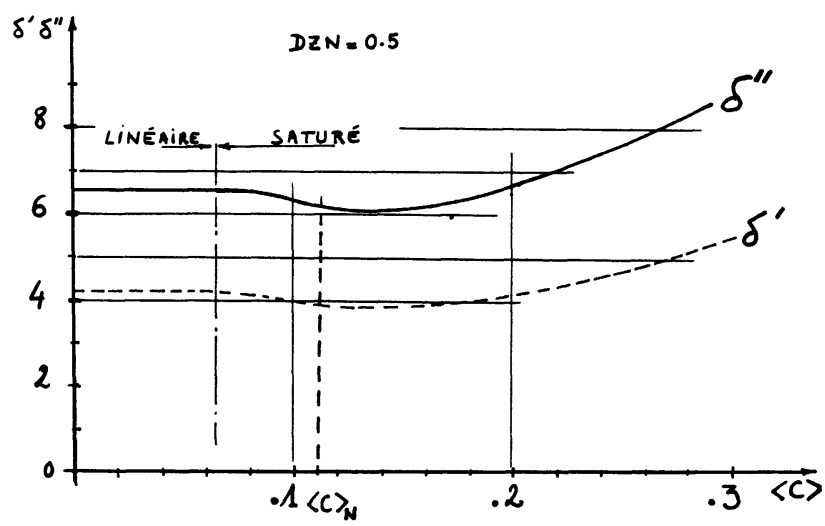

Fig. 3.3. - Influence de la saturation sur les facteurs de dimensionnement en régime non excité.

[Influence of the saturation on the S.P.R. in unexcited mode.]

Comme nous l'avons dit au $\$ 2.4$, dans une machine classique, si l'on demande transitoirement deux fois la puissance nominale, tous les paramètres 'de réglage étant conservés, il faut doubler la puissance de dimensionnement de l'alimentation. Le facteur de dimensionnement n'a donc pas varié en fonction de la puissance et, en particulier, en fonction du couple. Or dans une machine à réluctance variable, il n'en est rien en très forte saturation où le facteur de dimensionnement croît. Si la machine n'est pas conçue pour être très saturée, comme c'est le cas du prototype polydiscoïde de $200 \mathrm{~kW}$, la saturation a un effet favorable au couple nominal. La figure 3.3 nous en donne un exemple. Le couple nominal normalisé vaut 0,11 . A titre indicatif, le couple maximal que l'on pourrait obtenir en zone linéaire vaut 0,08 d'après (2.30) et le couple maximal convertible vaut 0,48 d'après (2.31).

On peut, dès lors, dire qu'en régime non excité, il sera très important pour dimensionner le convertisseur de connaître le couple maximal que devra fournir la machine ainsi que, bien sûr, sa fréquence maximale de rotation.

Le régime excité permet d'améliorer encore ces résultats si l'on profite de la saturation. Les ampères-tours d'excitation doivent alors « déplacer » le cycle de fonctionnement en zone saturée. Il est alors tout à fait possible d'atteindre des valeurs de $\delta_{01}^{\prime \prime}$ (convertisseur d'alimentation seul en mode 1 , voir \$ 2.3) inférieures à 2 (donc meilleures que dans les machines classiques à courant alternatif) et de $\delta_{02}^{\prime \prime}$ inférieures à 4 . La figure 3.4 nous donne un exemple en mode 2 , nous y voyons aussi l'influence du couple demandé dans l'exemple du prototype de $200 \mathrm{~kW}$.

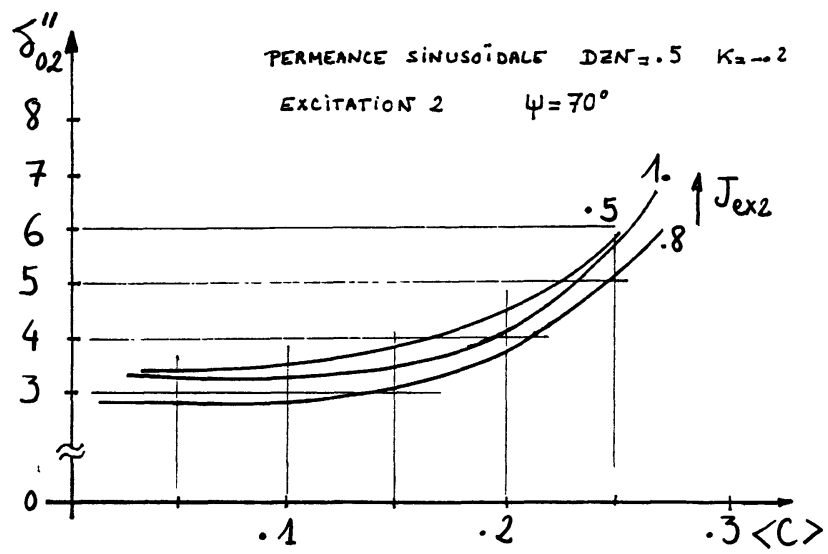

Fig. 3.4. - Facteur de dimensionnement en régime excité mode 2 .

[S.P.R. in second type excited mode.]

\subsection{CONSÉQUENCES DE L'ALIMENTATION OPTIMALE SUR LE COUPLE ÉLECTROMAGNÉTIQUE.}

\subsubsection{Expression du couple moyen en régime linéaire.}

* Machine non excitée.

En régime linéaire et avec une alimentation optimale (Fig. 3.1a), le couple moyen normalisé (2.28) s'exprime, d'après (2.4) et (2.5) de la façon suivante :

$$
\begin{array}{r}
\langle C\rangle=\left(\frac{U_{M}}{\omega}\right)^{2} \cdot \frac{1}{2 \pi}\left[\int_{-\psi}^{2 D \theta-\psi} \frac{\theta+\psi}{z(\theta)} \times\right. \\
\left.\times \mathrm{d} \theta-2 D \theta \int_{D \theta-\psi}^{2 D \theta-\psi} \frac{\mathrm{d} \theta}{z(\theta)}\right]
\end{array}
$$

où $D \theta=\pi-\theta_{0} / 2$ est l'angle de durée d'un palier de tension.

A angle interne et angle mort $\boldsymbol{\theta}_{0}$ fixés, le couple moyen normalisé $\langle C\rangle$ est proportionnel au carré du rapport $U_{\mathrm{M}} / \omega$ (ou encore au carré du flux maximum). On peut montrer qu'en régime linéaire ou saturé, pourvu que la fonction spatiale de la perméance soit paire, il existe par rapport à l'angle interne la symétrie moteur-générateur suivante (à $\theta_{0}$ fixé) :

$$
\langle C\rangle(-\psi)=-\langle C\rangle\left(\psi-\theta_{0}\right)
$$

$\left(\right.$ si $\left.\theta_{0}=0:\langle C\rangle(-\psi)=-\langle C\rangle(\psi)\right)$.

Cette condition de parité de $z(\theta)$ est pratiquement toujours respectée. Les facteurs de dimensionnement en fonctionnement génératrice satisfont à la même règle de symétrie et peuvent donc se déduire $\mathrm{du}$ fonctionnement moteur. Un exemple de cette symétrie est donné à la figure 3.5 . 


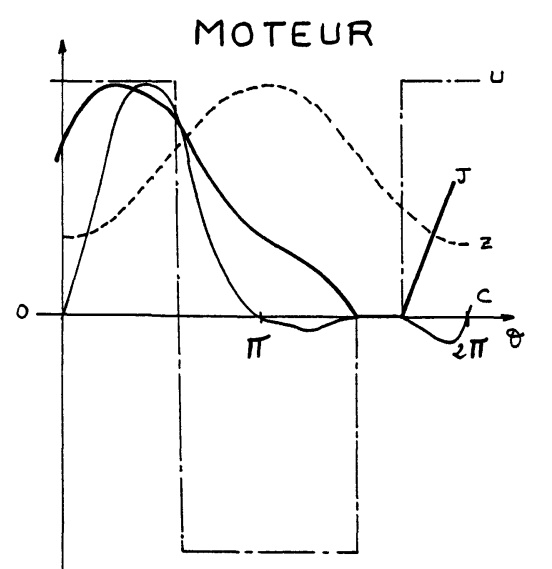

$\psi=70^{\circ} \quad \theta_{0}=20^{\circ}$

$\frac{U_{M}}{\omega}=0,32$

$J_{M}=1$

$\langle c\rangle=0,052$

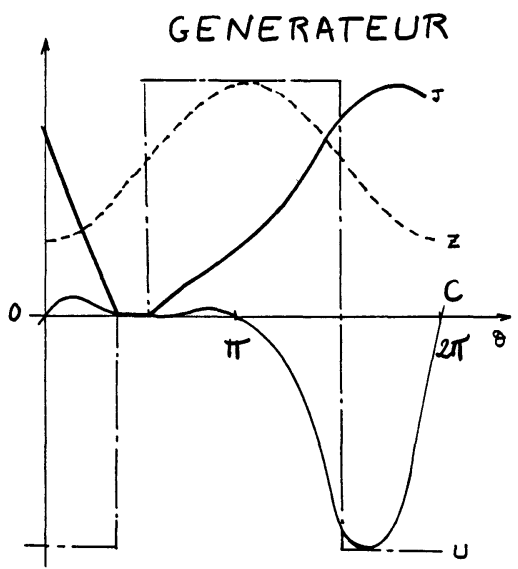

$\psi=-90^{\circ} \quad \theta_{0}=20^{\circ}$

$\frac{U_{M}}{\omega}=0,32$

$J_{H}=1$.

$\langle c\rangle=-.052$

permeance sinusoidale DZN $=.5$

Fig. 3.5. - Symétrie des fonctionnements moteur et générateur en régime non excité.

[Symmetry of motor and generator functions in unexcited mode.]

Dans le cas particulier d'une onde spatiale de réluctance sinusoïdale et d'un angle $\theta_{0}$ nul (cas très souvent optimal), le couple moyen vaut :

$$
\langle C\rangle=\left(\frac{U_{\mathrm{M}}}{\omega}\right)^{2} \cdot D R N \cdot \sin \psi
$$

où

$$
D R N=D Z N /\left(1-D Z N^{2}\right) .
$$

Dans un cas général, la forme de la caractéristique couple fonction de l'angle interne est plus ou moins proche d'une sinusoïde (3.3) selon la forme de l'onde spatiale de perméance.

* Machine excitée.

Le calcul est ici beaucoup plus complexe si l'on souhaite exprimer le couple moyen en fonction des ampères-tours d'excitation et non du flux moyen. Nous ne donnerons que le résultat approché concernant une onde sinusoïdale de réluctance dans le cas du mode 1. En alimentant la machine de la manière décrite à la figure 3.1b ( $\varphi_{0}$ est le flux moyen) on obtient :

$$
\langle C\rangle=\frac{2}{\pi} \frac{U_{\mathrm{M}}}{\omega} \cdot \varphi_{0} \frac{D Z N}{1-D Z N^{2}} \cdot \sin \psi
$$

où

$$
\varphi_{0}=\left(J_{\text {ex } 1}-\frac{2}{\pi} \frac{U_{\mathrm{M}}}{\omega} D Z N \cdot \cos \psi\right)\left(1-D Z N^{2}\right)
$$

en faisant l'approximation suivante (justifiée dans les cas optimaux) :

$$
\varphi_{0} \simeq J_{\mathrm{ex} 1} \cdot\left(1-D Z N^{2}\right)
$$

l'expression approchée du couple devient :

$$
\langle C\rangle \simeq \frac{2}{\pi} D Z N \frac{U_{\mathrm{M}}}{\omega} J_{\mathrm{ex} 1} \sin \psi .
$$

En régime linéaire, le couple moyen est sensiblement proportionnel au rapport $\frac{U_{\mathrm{M}}}{\omega}$ et aux ampèrestours d'excitation. Dans un cas plus général, il suffit que l'expression de l'onde spatiale de perméance (ou de réluctance) soit paire pour avoir la symétrie moteur-générateur suivante :

$$
\langle C\rangle(-\psi)=-\langle C\rangle(\psi) .
$$

3.3.2 Influence des paramètres de l'alimentation sur le couple. - L'étude de l'influence des paramètres de l'alimentation $\left(\frac{U_{\mathrm{M}}}{\omega}, \psi\right.$ et éventuellement les ampères-tours d'excitation) sur le couple ne peut se faire que pour une machine donnée. Dans des machines de caractéristiques différentes de l'exemple présenté, l'allure générale des courbes est conservée. Les résultats quantitatifs ne peuvent ainsi être obtenus que sur des données précises. Nous choisissons les données du prototype $200 \mathrm{~kW}(D Z N=0,5$ et $K=-0,2)$, sur chaque courbe la forme de l'onde spatiale de perméance du modèle est précisée.

* En régime non excité.

La figure 3.6 montre l'influence de l'angle interne sur le couple moyen pour quelques formes d'onde de perméance (le circuit magnétique est saturé dès que le couple moyen normalisé dépasse 0,06 ). On retrouve l'allure prévue, analytiquement au § 3.3.1.

La figure 3.7a donne la variation de l'ondulation relative du couple monophasé lorsque l'angle interne varie, on comprend aisément que pour limiter les pulsations du couple, il faut travailler avec un angle d'avance à l'opposition compris entre 40 et $70^{\circ}$. Si l'on observe alors l'évolution du facteur de dimensionnement $\delta^{\prime \prime}$ en fonction de $\psi$ (cette évolution est d'ailleurs la même pour $\delta^{\prime}$ ), on constate que l'optimum se situe aussi dans la même zone (Fig. 3.7b). Il est alors parfaitement possible d'obtenir un bon compromis, entre une faible pulsation du couple monophasé et un bon facteur de dimensionnement 


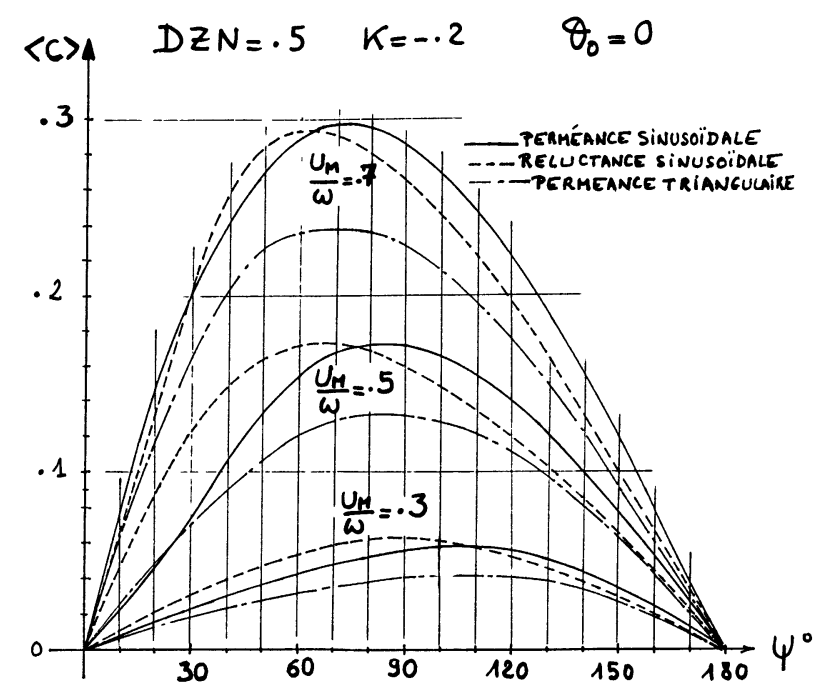

Fig. 3.6. - Influence de l'angle interne sur le couple moyen pour quelques formes de l'onde spatiale de perméance.

[Influence of the internal angle of the mean torque for various shapes of the spatial wave of permeance.]

du convertisseur, dans le cas des machines multimonophasées.

Dans le cas d'une machine triphasée, il est intéressant d'observer l'influence de l'angle interne sur l'harmonique 3 qui ne s'annule pas par composition des couples monophasés. Les calculs montrent que les valeurs optimales de $\psi$ sont comprises entre 30 et $50^{\circ}$, l'harmonique de rang 6 est en général très inférieur à celui de rang 3 .

Enfin, la figure 3.8 nous montre, pour quelques valeurs de l'angle interne, l'évolution du couple moyen en fonction du rapport $\frac{U_{\mathrm{M}}}{\omega}$ proportionnel au flux maximal. On y a superposé la courbe hypothétique obtenue en l'absence de saturation.

* En régime excité.

Dans le cas du régime excité, l'influence de la saturation est fondamentale. La valeur des ampèrestours d'excitation et l'allure de la caractéristique magnétique (valeur de $K$ en particulier) sont d'une grande importance. Pour ne pas alourdir l'article, nous n'en présentons que quelques courbes.

Les figures 3.9 et 3.10 donnent respectivement, en fonction de l'angle interne, l'évolution du couple moyen et du facteur de dimensionnement $\delta_{02}^{\prime \prime}$ (convertisseur d'alimentation en courant unidirectionnel). Ceci correspond à l'exemple de la machine polydiscoïde de $200 \mathrm{~kW}$.

On observe ici encore, des valeurs optimales de $\psi$ se situant aux alentours de $60^{\circ}$. Un compromis entre un faible facteur de dimensionnement et un couple pulsatoire minimal peut être trouvé selon la priorité accordée au dimensionnement mécanique ou à celui du convertisseur.

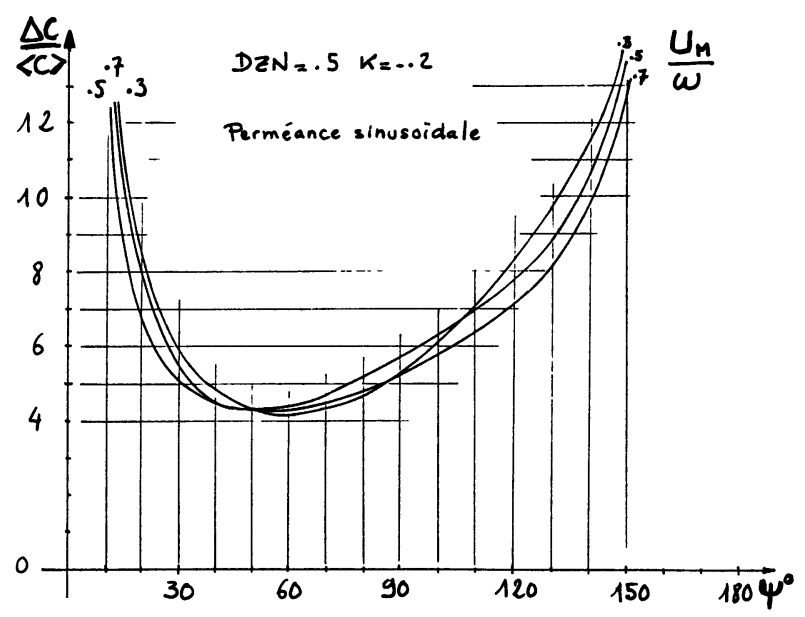

a)

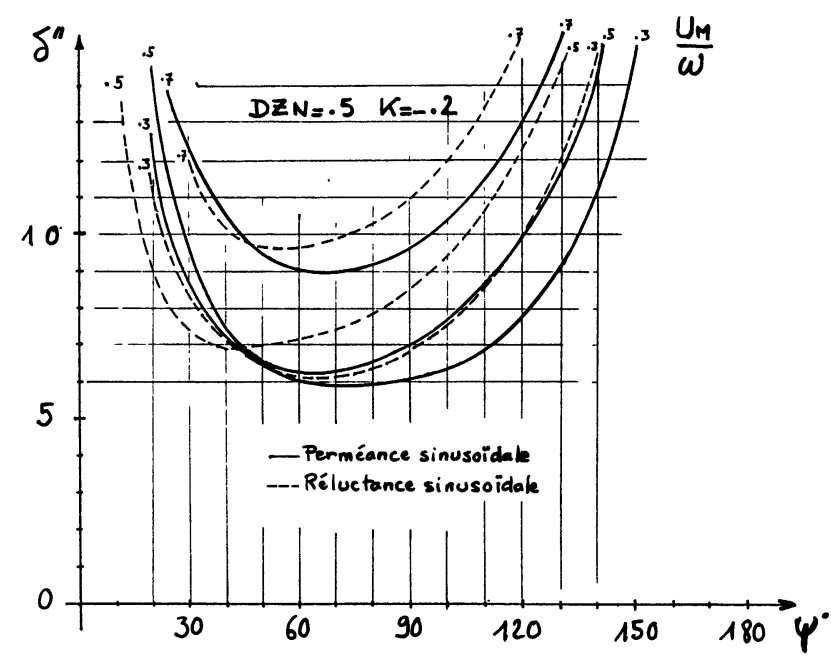

b)

Fig. 3.7. - Influence de l'angle interne. 3.7a: sur l'ondulation du couple, $3.7 \mathrm{~b}$ : sur le facteur de dimensionnement.

[Influence of the internal angle. 3.7a: on the torque ondulation, $3.7 \mathrm{~b}$ : on the S.P.R.]

A la figure 3.11 , on remarque que le couple moyen croît à peu près linéairement en fonction de $U_{\mathrm{M}} / \omega$ lorsque l'angle interne et les ampères-tours d'excitation sont figés, c'est ce qu'exprimait la formule 3.5 définie en régime non saturé. L'ondulation relative du couple est d'autant plus grande que le flux maximal est faible ce qui s'explique par les faibles variations des ampères-tours d'alimentation pour des ampères-tours d'excitation fixés. Dans ces conditions le couple est particulièrement pulsatoire (Fig. 3.12).

Une ondulation du couple importante est à prohiber. Aussi l'alimentation des machines excitées multimonophasées de forte puissance doit se faire à faible excitation pour les faibles couples et à excitation maximale pour les forts couples afin de conserver le dimensionnement minimal du convertisseur prévu pour la vitesse et le couple maximaux. 


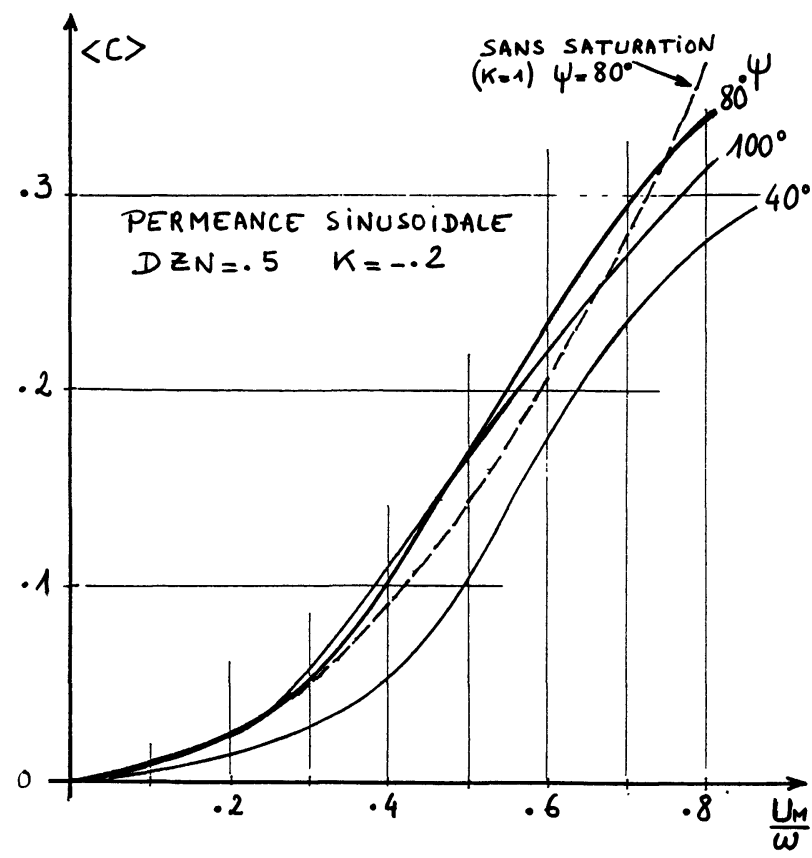

Fig. 3.8. - Couple moyen en fonction du rapport $U_{\mathrm{M}} / \omega$ à angle fixé.

[Mean torque vs. $U_{\mathrm{M}} / \omega$ for given internal angle.]

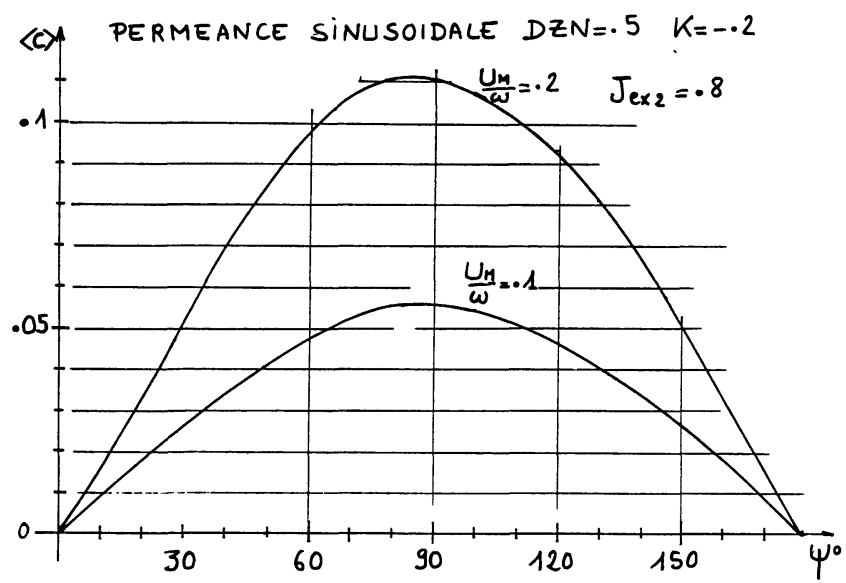

Fig. 3.9. - Couple moyen en régime excité en fonction de l'angle interne.

[Mean torque in excited mode vs. internal angle.]

\subsection{INFLUENCE DES CARACTÉRISTIQUES DE LA MACHINE.}

* En régime non excité.

La forme de l'onde spatiale de perméance présente une influence relativement réduite en ce qui concerne les facteurs du dimensionnement, elle affecte surtout le couple instantané. L'ondulation relative $\Delta C /\langle C\rangle$ et l'harmonique 3 en particulier $y$ sont très sensibles. Les figures $3.13 \mathrm{a}$ et $\mathrm{b}$ donnent, en l'absence de saturation, l'ondulation relative et le troisième harmonique du couple monophasé, obte-

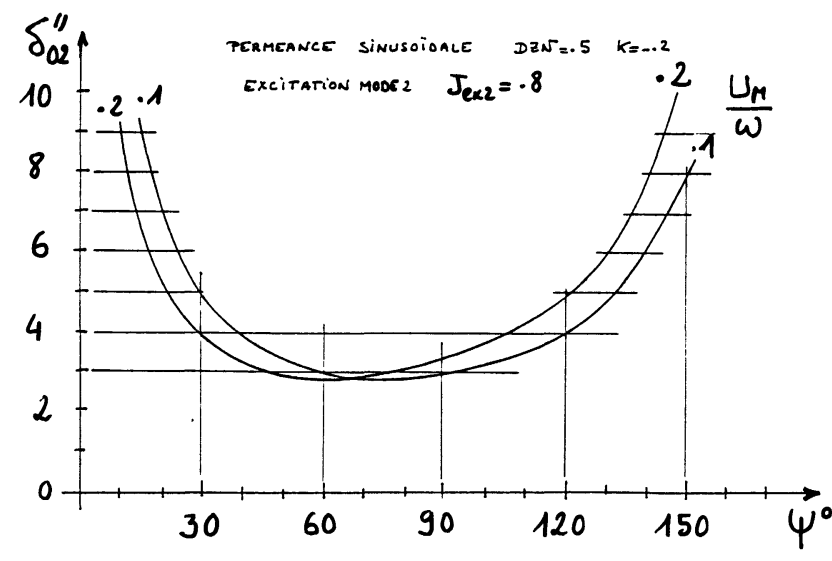

Fig. 3.10. - Facteur de dimensionnement $\delta_{02}^{\prime \prime}$ (en courant unidirectionnel) en fonction de l'angle interne.

[S.P.R. $\delta_{02}^{\prime \prime} v s$. internal angle.]

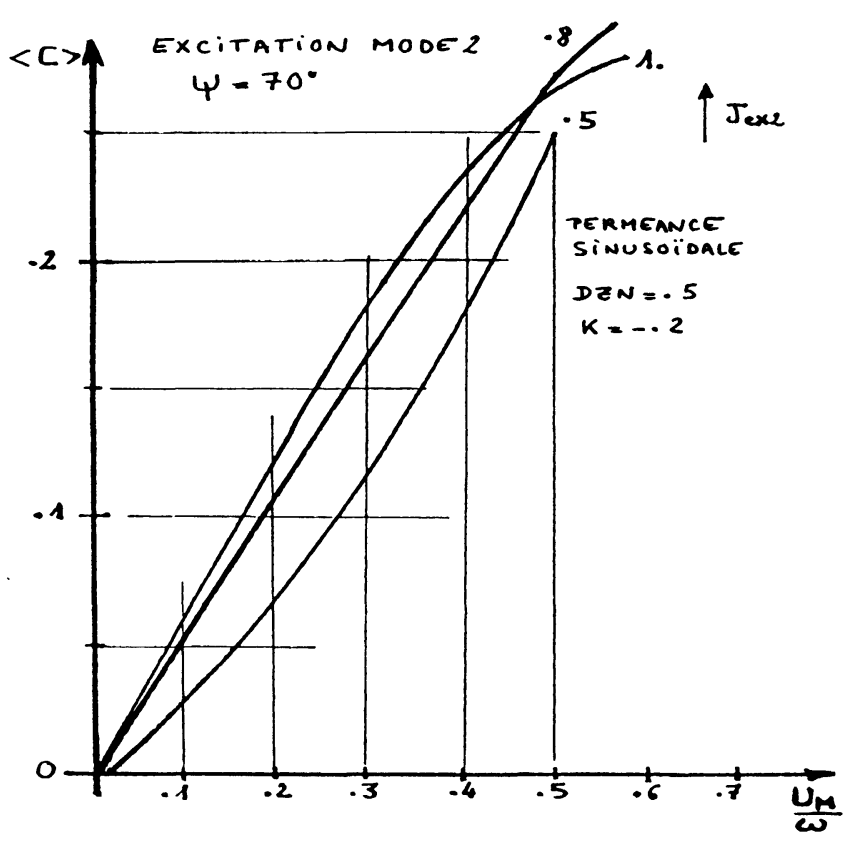

Fig. 3.11. - Couple moyen en fonction du rapport $U_{\mathrm{M}} / \omega$.

[Mean torque vs. $U_{\mathrm{M}} / \omega$ ratio.]

nus en optimisant $\delta^{\prime \prime}$, en fonction de la variation relative de perméance $D Z N$ pour quelques formes de la variation de la perméance (la forme trapézoïdale correspond à un angle de variation de $2 \pi / 3$ et à un "plat » à l'opposition de $\pi / 2$ ).

En régime saturé, ces grandeurs diminuent ou augmentent selon les valeurs de $D Z N$, de $K$ et du couple demandé (niveau de saturation atteint). Il est difficile de donner une conclusion générale à ce sujet. Cependant, suite à de nombreux calculs, il semble que le meilleur comportement en saturation soit obtenu avec l'onde spatiale de perméance sinusoïdale.

La valeur de $K$ qui définit la caractéristique de la 


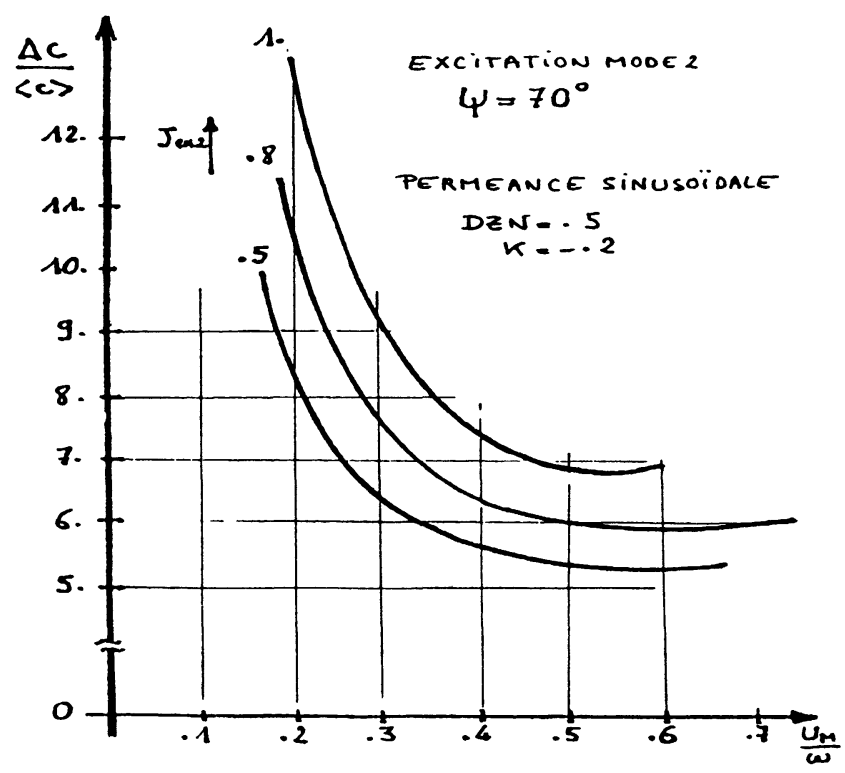

Fig. 3.12. - Ondulation du couple en fonction du rapport.

[Torque undulation $v s . U_{\mathrm{M}} / \omega$ ratio.]

machine en zone saturée est d'une grande importance en ce qui concerne les facteurs de dimensionnement. Rappelons que pour des machines fonctionnant en saturation $K$ est négatif ou nul (voir $\S 2.5$, Fig. 2.6), et plus $K$ est négatif, plus on approche rapidement la limite du couple maximal convertible (Eq. (2.31)) et donc plus les facteurs de dimensionnement se dégradent rapidement. C'est ce que montre la figure 3.14 .

Une valeur de $K$, réaliste, particulièrement intéressante est la valeur zéro. On remarque alors que la saturation peut être très profitable puisqu'elle permet une diminution considérable du facteur de dimensionnement surtout lorsque la variation relative de la perméance $D Z N$ est grande.

\section{* En régime excité.}

En régime linéaire, les calculs numériques (et analytiques, voir § 3.5) montrent que les valeurs élevées de la variation relative de perméance $D Z N$ ne sont pas favorables aux facteurs de dimensionnement (contrairement au cas du régime non excité) sauf dans le cas d'une variation sinusoïdale de réluctance et d'un cas particulier de perméance trapézoïdale, c'est ce que montre la figure 3.15 dans le cas où l'optimisation est effectuée avec $\alpha=0,2$ (coefficient de pondération des ampères-tours d'excitation, voir § 2.3).

En régime linéaire, une onde sinusoïdale de réluctance nous permet d'atteindre une valeur de $\delta_{01}^{\prime \prime}$ (en courant bidirectionnel) égale à $\pi / 2$ soit 1,57 avec un troisième harmonique du couple quasiment inexistant et, une onde triangulaire de perméance permet d'atteindre un facteur de dimensionnement $\delta_{01}^{\prime \prime}$ unitaire $\left(\delta_{02}^{\prime \prime}=2\right)$ avec des harmoniques de rang REVUE DE PHYSIQUE APPLIQUÉE. - T. 22, N* 5, MAI 1987

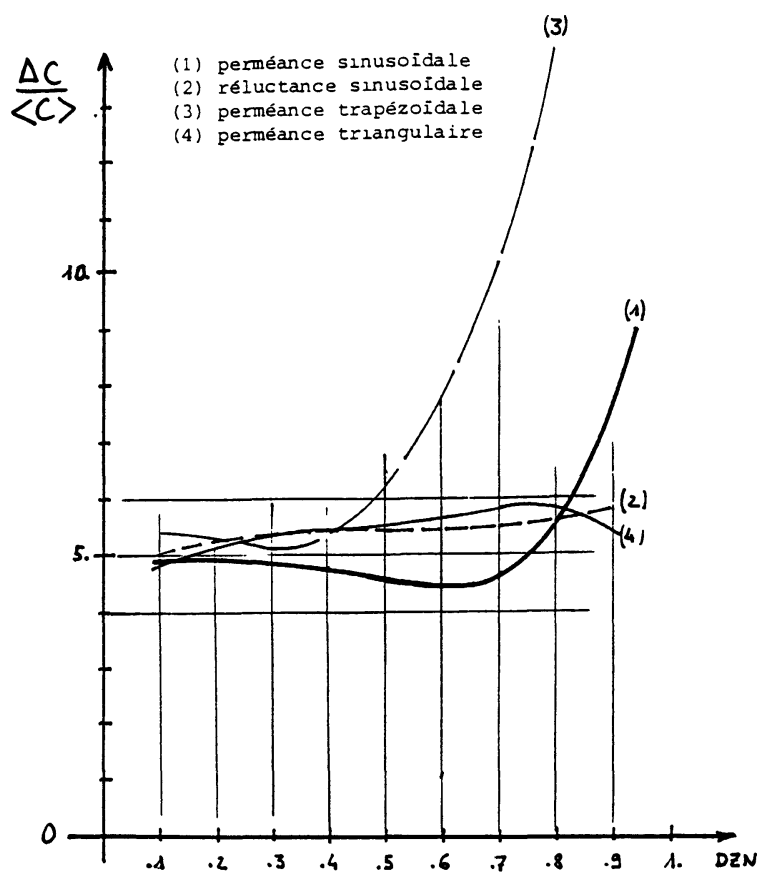

a)

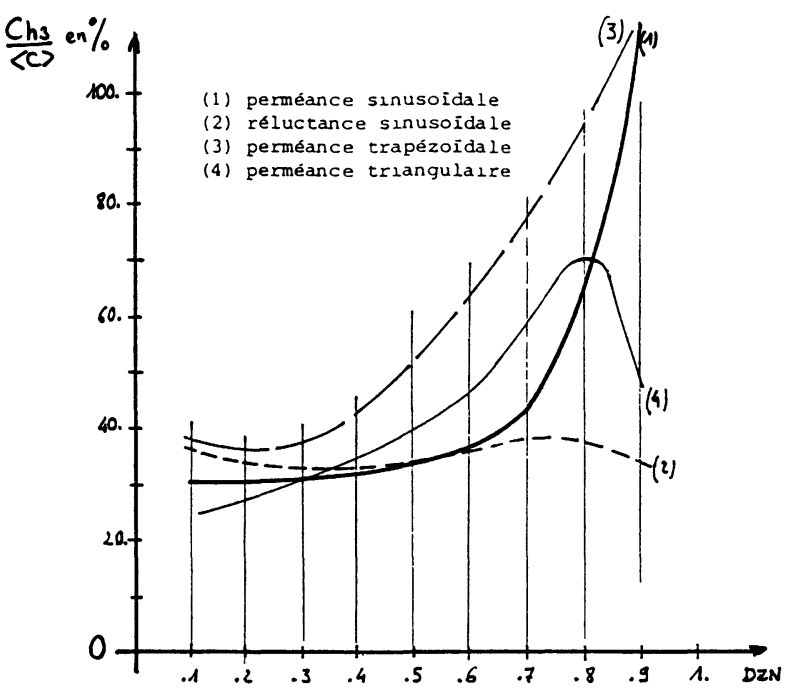

h)

Fig. 3.13. - Influence de la forme de l'onde spatiale et de la variation relative de la perméance, en régime linéaire et non excité. 3.13a: sur l'ondulation relative du couple monophasé, $3.13 \mathrm{~b}$ : sur le troisième harmonique du couple.

[Influence of the shape of the spatial wave and of the relative variation of permeance in non excited mode. $3.13 \mathrm{a}$ : on the relative undulation of the one phase torque. $3.13 \mathrm{~b}:$ on the third harmonic of the torque.]

pair du couple quasi nuls à condition d'avoir de faibles valeurs de la variation relative de la perméance $D Z N$. Ces résultats très intéressants sont obtenus aux dépens de l'ondulation relative du couple monophasé. La saturation permet, tout en conservant les qualités de bon facteur de dimension- 

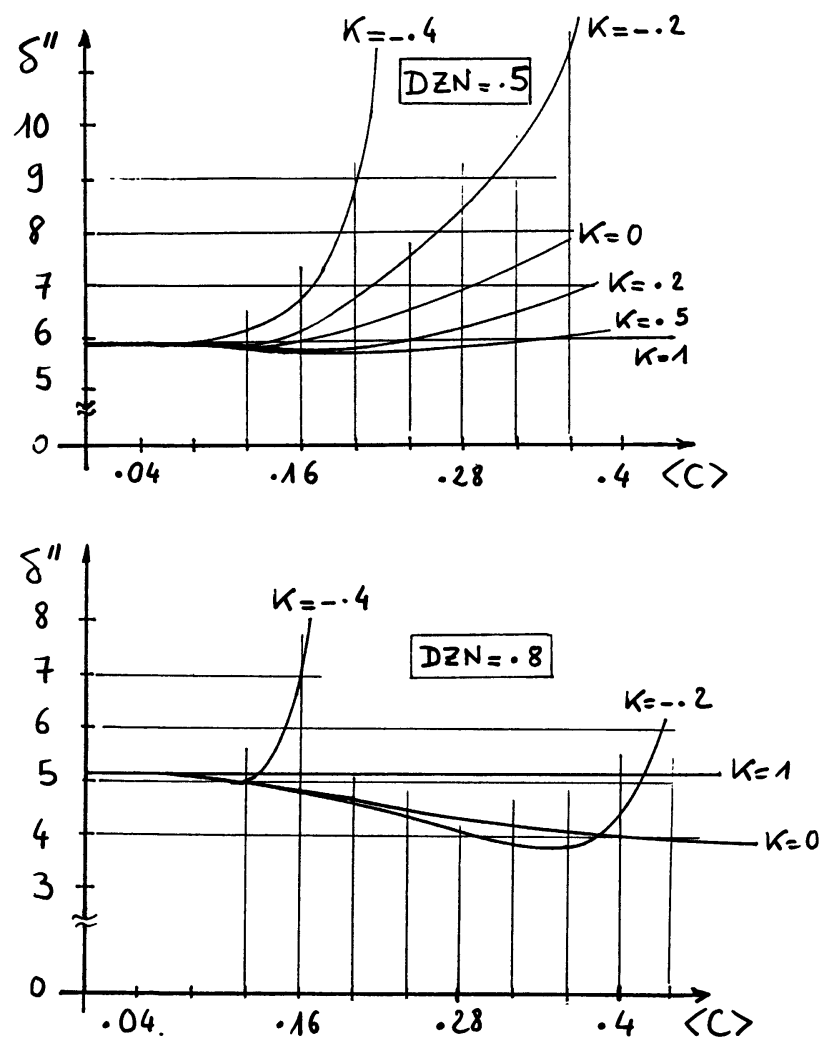

Fig. 3.14. - Influence du paramètre $K$ (saturation) sur l'évolution du facteur de dimensionnement en fonction du couple demandé dans le cas d'une onde sinusoïdale de perméance.

[Influence of the parametre $K$ (saturation) on the evolution of the S.P.R. vs. torque (sine wave of permeance).]

nement du régime linéaire, de diminuer très sensiblement l'ondulation relative et les harmoniques du couple à couple moyen donné. Mais les facteurs de dimensionnement subissent, comme en régime non excité, une dégradation lorsque le couple demandé augmente.

Comme en régime non excité, plus le paramètre $K$ est voisin de 0 , plus les harmoniques du couple sont faibles et moins vite se dégradent les facteurs de dimensionnement.

Lorsque les machines sont saturées, il est particulièrement intéressant d'avoir une variation relative de perméance $D Z N$ élevée. Dans ces conditions, il est tout à fait possible d'obtenir des facteurs de dimensionnement $\delta_{02}^{\prime \prime}$ inférieur à 3 (courant unidirectionnel) et $\delta_{01}^{\prime \prime}$ inférieur à 1,5 (courant bidirectionnel). Ces valeurs sont à comparer avec celles des machines classiques à courant alternatif qui sont supérieures ou égales à 2).

En ce qui concerne la forme optimale de l'onde spatiale de perméance, la forme sinusoïdale satisfait, en général, à la fois au critère de faible dimensionnement et aux critères de couple peu pulsatoire et peu ondulé en triphasé. La forme triangulaire s'est révélée tout particulièrement intéressante dans un

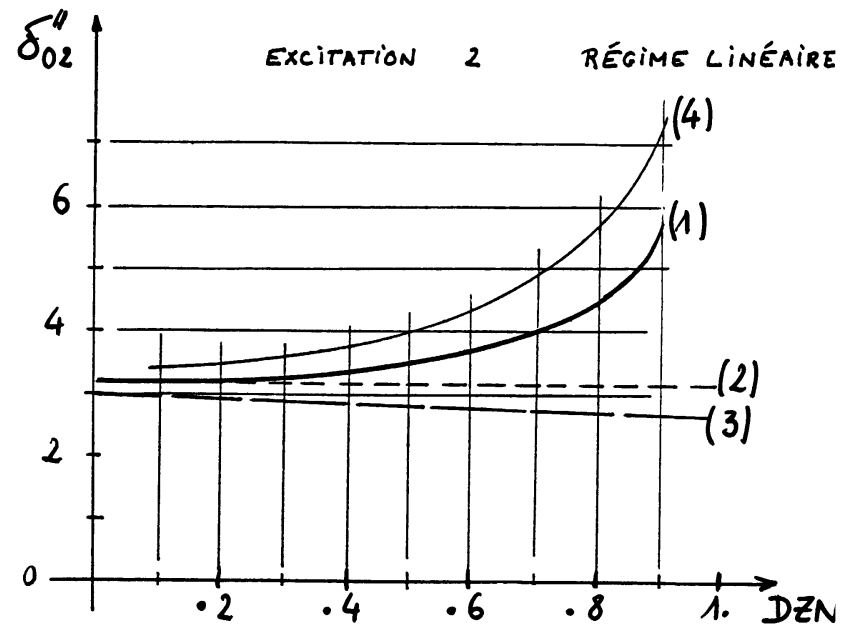

(1) perméance sınusoidale

(2) réluctance sinusoidale

(3) perméance trapézoidale

(4) perméance triangulaire

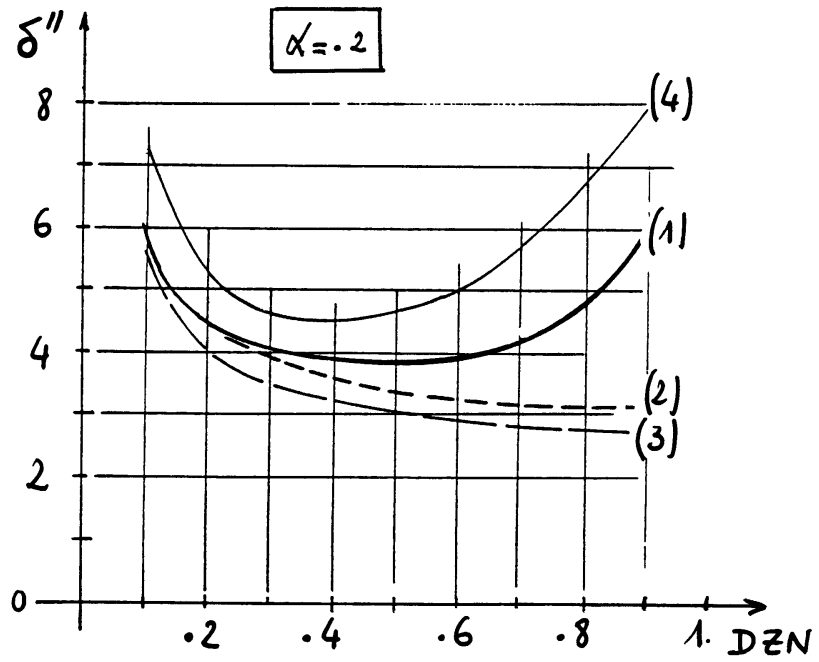

Fig. 3.15. - Facteurs de dimensionnement du convertisseur d'alimentation seul $\delta_{02}^{\prime \prime}$ et de l'ensemble convertisseurs d'alimentation et d'excitation $\delta^{\prime \prime}$ optimisés avec $\alpha=0,2$ pour différentes formes de l'onde de perméance en régime linéaire.

[S.P.R. of the convertor taken in isolation $\delta_{02}^{\prime \prime}$ and of both the supply and excitation convertors $\delta^{\prime \prime}(\alpha=0.2)$ for various waves of permeance in linear area.]

cas très particulier de caractéristiques magnétiques et d'alimentation ( $K=0$ et $\left.U_{\mathrm{M}} / \omega=\mathrm{Cte}\right)$, il est alors possible d'atteindre un facteur de dimensionnement en courant bidirectionnel $\delta_{01}^{\prime \prime}$ unitaire et une machine tétraphasée (ou diphasée) à couple quasiment constant. Nous montrons au paragraphe suivant comment ces résultats remarquables peuvent être obtenus.

3.5 LIMITE ABSOLUE DES FACTEURS DE DIMENSIONNEMENT. - Soit une machine à réluctance variable non saturée et à variation triangulaire de perméance. 
Elle est excitée et alimentée en tension carrée donc par un flux triangulaire décalé de l'angle interne $\psi$ par rapport à l'onde de perméance (Fig. 3.1b, § 3.1). Les expressions de $Z(\theta)$ et $\varphi(\theta)$ sont :

$$
\begin{aligned}
& \forall \theta \in[0, \pi] Z(\theta)=1+D Z N \cdot \frac{2 \theta-\pi}{\pi} \\
& \forall \theta \in[\pi, 2 \pi] Z(\theta)=1+D Z N \frac{-2 \theta+3 \pi}{\pi} \\
& \forall \theta \in[-\psi, \pi-\psi] \varphi(\theta)= \\
& =\varphi_{0}+\varphi_{M} \frac{2 \theta-\pi+2 \psi}{\pi}
\end{aligned}
$$

$\forall \theta \in[\pi-\psi, 2 \pi-\psi] \varphi(\theta)=$

$$
=\varphi_{0}+\varphi_{M} \cdot \frac{-2 \theta+3 \pi-2 \psi}{\pi} \text {. }
$$

Si $\psi=0$ et si $\varphi_{\mathrm{M}} / \varphi_{0}=D Z N$, les fonctions $Z(\theta)$ et $\varphi(\theta)$ sont homothétiques et par conséquent les ampères-tours (2.7) sont constants mais le couple moyen est nul. Maintenant, décalons $\varphi(\theta)$ d'un angle $\psi$ faible (Fig. 3.16). On obtient des ampères-

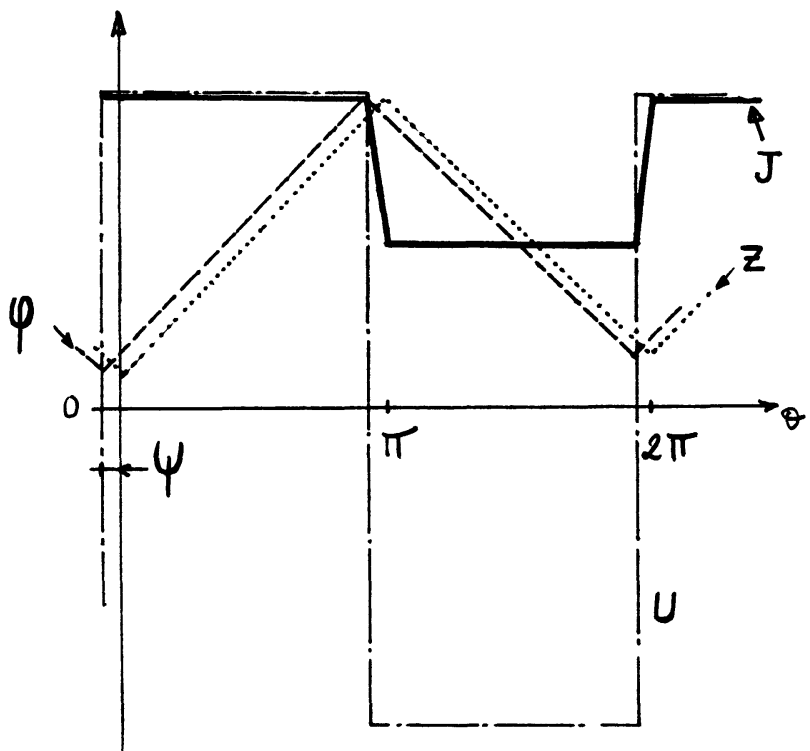

Fig. 3.16. - Alimentation optimale conduisant au facteur de dimensionnement minimal à condition d'avoir une variation triangulaire de perméance.

[Optimal supply to reach a minimum S.P.R. with a triangular variation of permeance.]

tours en créneaux de faible amplitude et un couple moyen non nul en respectant les conditions suivantes :

$$
\varphi_{0} \gg \varphi_{M} \cdot \frac{2 \psi}{\pi} \quad \text { et } \quad \frac{\varphi_{M}}{\varphi_{0}}=D Z N
$$

Ces deux conditions réunies imposent : $D Z N \ll 2 \psi / \pi$.
Alors en mode d'excitation 1 , on a les valeurs suivantes des ampères-tours d'alimentation et d'excitation :

$$
J_{\mathrm{ex} 1}=\varphi_{0} \quad \text { et } \quad J_{\mathrm{aM} 1}=\varphi_{\mathrm{M}} \cdot \frac{2 \psi}{\pi}
$$

Le couple vaut :

$$
\begin{gathered}
\langle C\rangle=\frac{4 \cdot D Z N^{2}}{\pi} \cdot J_{\mathrm{ex} 1} \cdot \frac{\psi}{\pi} \\
\frac{\Delta C}{\langle C\rangle}=\frac{\pi}{2 \cdot \psi \cdot D Z N} .
\end{gathered}
$$

Les facteurs de dimensionnement atteignent alors le minimum théorique :

$$
\delta_{01}^{\prime}=\delta_{01}^{\prime \prime}=1
$$

( $\delta_{02}^{\prime}=\sqrt{2}$ et $\delta_{02}^{\prime \prime}=2$ en courant bidirectionnel).

Les conditions sont, très restrictives (valeur de $D Z N$ très faible, ampères-tours d'excitation très élevés...) et le couple obtenu est très faible et très pulsatoire. Montrons maintenant que la saturation (avec une valeur de $K$ nulle (Fig. 2.6) permet de limiter les restrictions et d'obtenir des résultats applicables pratiquement.

Le cycle de fonctionnement est supposé être totalement situé en zone saturée $(J \geqslant 1)$. Le flux et le couple normalisés calculés à patir du modèle à 3 pentes s'expriment de la manière suivante :

$$
\begin{gathered}
\varphi(\theta)=Z(\theta)+[K M Z+K(Z(\theta)-1)] \times \\
\times[J(\theta)-1] \\
C(\theta)=\frac{\mathrm{d} Z(\theta)}{\mathrm{d} \theta} \cdot\left[(1-K) \cdot\left(J-\frac{1}{2}\right)+K \cdot \frac{J^{2}}{2}\right]
\end{gathered}
$$

où

$$
K M Z=(K-1) D Z N+1
$$

alors les ampères-tours valent :

$$
J(\theta)=1+\frac{\varphi(\theta)-Z(\theta)}{K M Z+K(Z(\theta)-1)} .
$$

Si $K=0$, il suffit de respecter la relation $\varphi_{\mathrm{M}}=$ $D Z N$ et que l'angle interne $\psi$ soit très inférieur à $\pi$ (on montre, numériquement, que $\psi=\pi / 4$ est une limite acceptable) pour avoir des ampères-tours en créneaux déphasés de $\psi$ par rapport à la tension :

$\forall \theta \in[-\psi, \pi-\psi] J(\theta)=\frac{\varphi_{0}+\varphi_{M}\left(2 \frac{\psi}{\pi}-1\right)}{1-\varphi_{M}}$

$\forall \theta \in[\pi-\psi, 2 \pi-\psi] J(\theta)=\frac{\varphi_{0}-\varphi_{M}\left(2 \frac{\psi}{\pi}+1\right)}{1-\varphi_{M}}$ alors :

$$
J_{\mathrm{ex} 1}=\frac{\varphi_{0}-\varphi_{\mathrm{M}}}{1-\varphi_{\mathrm{M}}} \quad \text { et } \quad J_{\mathrm{aM} 1}=\frac{\varphi_{\mathrm{M}}}{1-\varphi_{\mathrm{M}}} \frac{2 \psi}{\pi} .
$$


Pour satisfaire à la condition de fonctionnement en zone saturée, il faut :

$$
J_{\text {ex1 }}-2 J_{\text {aM1 }} \geqslant 1
$$

(ou en mode 2: $J_{\mathrm{ex} 2}+J_{\mathrm{aM} 2} \geqslant 1$ ) qui devient :

$$
\varphi_{0} \geqslant 1+D Z N .2 \psi / \pi \text {. }
$$

Le couple moyen s'exprime par:

$$
\langle C\rangle=\frac{4 \cdot D Z N^{2}}{1-D Z N} \cdot \frac{\psi}{\pi^{2}}
$$

et les facteurs de dimensionnement $\delta_{01}^{\prime}$ et $\delta_{01}^{\prime \prime}$ restent unitaires.

Cette expression nous montre que plus la variation relative de perméance $D Z N$ est grande, plus on peut occuper une proportion importante de l'aire maximale du cycle (voir $\S 2.5$, expressions (2.30) et (2.31)) à angle interne $\psi$ donné. En fait pour conserver les facteurs de dimensionnement optimaux, il est nécessaire d'avoir un angle interne faible (inférieur à $30^{\circ}$ environ). A angle interne donné, une machine dont la valeur de $D Z N$ vaut 0,8 permet de "remplir le cycle " quatre fois mieux que si $D Z N$ valait 0,5 (expression (3.12)).

Pour minimiser les ampères-tours d'excitation et l'ondulation du couple, on choisit un fonctionnement en zone saturée à la limite de la zone linéaire. Le mode d'excitation 2 se prête bien à la réalisation de cette condition. En effet, des ampères-tours d'excitation $J_{\text {cx2 }}$ égaux aux ampères-tours de saturation permettent de maintenir le cycle de fonctionnement en zone saturée à la limite du régime linéaire quel que soit le flux maximal d'alimentation. Alors, l'ondulation du couple monophasé vaut :

$$
\frac{\Delta C}{\langle C\rangle}=\left[1+D Z N\left(\frac{4 \psi}{\pi}-1\right)\right] \cdot \frac{\pi}{\psi} .
$$

Une telle machine devra donc être alimentée à flux maximal constant $\left(U_{\mathrm{M}} / \omega=\right.$ Cte $)$ tel que le flux maximal normalisé soit égal à $D Z N$, la variation du couple pourra alors être obtenue par réglage de l'angle interne [7].

Les harmoniques du couple de rang pair sont alors très faibles, il faut donc choisir une machine diphasée ou tétraphasée pour obtenir un couple constant.

Cette propriété des machines excitées à variation de perméance triangulaire peut, dans un cas très particulier, s'appliquer à une machine non excitée très saturée. En effet, si $D Z N$ tend vers 1 (cycle $\varphi(j)$ très ouvert) et si $K=0$, on peut approcher les valeurs $\delta^{\prime \prime}=2$ et $\delta^{\prime}=\sqrt{2}$, le courant étant constitué de créneaux unidirectionnels, l'ondulation relative du couple monophasé vaut 2 ce qui est très faible comparé aux valeurs obtenues dans les machines excitées. Un nombre pair de phases permet, là aussi, d'obtenir un couple quasiment constant. De telles caractéristiques ne semblent pas utopiques. En effet, une machine de $5 \mathrm{~kW}$ de caractéristiques $D Z N=$ 0,92 et $K=0$ [9] a été construite, mais sa variation de perméance n'est pas triangulaire. Il semble qu'une recherche dans la forme et les proportions des motifs permette de réaliser les caractéristiques souhaitées. Des calculs de champs et des expériences ont déjà montré la faisabilité de la caractéristique $K=0$ très intéressante dans de nombreux cas.

3.6 CONCLUSION. - La recherche des formes d'ondes d'alimentation optimales des machines à réluctance variable a permis de diminuer considérablement le dimensionnement des convertisseurs en adoptant une source de tension en créneaux. De telles ondes peuvent être obtenues avec des structures classiques, très simples dans les cas des régimes non excités et excités mode 2 où un courant d'alimentation unidirectionnel suffit. Le gain obtenu sur le dimensionnement, par rapport aux alimentations en courant est surtout important si la variation relative de perméance $D Z N$ est supérieure à 0,5 .

Le régime excité, à condition de travailler en zone saturée, permet encore de diminuer le facteur de dimensionnement du convertisseur d'alimentation (dans un rapport 2 environ) par rapport au régime non excité. Mais il faut alors accepter un couple monophasé très pulsatoire qui peut être inadmissible dans les machines multimonophasées de forte puissance. Toutefois, l'ondulation du couple polyphasé est très réduite.

Il semble intéressant de concevoir simultanément la machine et son convertisseur car il existe des associations caractéristiques machines-convertisseurs particulièrement avantageuses. La forme des motifs, en particulier, est très influente sur la forme de l'onde spatiale de perméance, sur la variation relative de perméance et sur le facteur de saturation $K$.

Enfin, la prolongation de cette optimisation aux machines à phases couplées magnétiquement apportera un complément indispensable à l'étude des ensembles convertisseurs-machines à réluctance variable. Ce travail est actuellemement poursuivi au L.E.SI.R. à l'E.N.S. de Cachan.

\section{Annexe 1.}

EXEMPLES DE STRUCTURES OPTIMALES DE CONVERTISSEURS. - Dans une étude des différentes structures des convertisseurs susceptibles de générer les formes d'ondes optimales d'alimentation [7], on a déterminé le facteur de dimensionnement global de chaque ensemble. Selon qu'il s'agit de machines à phases électriquement non couplées ou couplées (étoile ou polygone), les formes d'ondes de tension s'éloignent plus ou moins des formes optimales. En régime excité et dans le cas des machines polyphasées, on s'est soucié des réactions du flux d'alimentation sur celui d'excitation. Pour éliminer ces réac- 
tions, il suffit que la somme des flux instantanés soit constante, dans ces conditions, les enroulements d'excitation de chaque phase connectés en série sont soumis à une force électromotrice de réaction nulle. Il est alors aisé d'alimenter le bobinage d'excitation en courant constant avec une source de tension.

Deux structures intéressantes sont présentées, l'une en régime non excité pour l'alimentation d'une machine à phases électriquement indépendantes (Fig. A1), l'autre en régime excité pour l'alimentation d'une machine triphasée couplée en étoile (Fig. A2).

Le facteur de dimensionnement global doit prendre en compte le nombre d'interrupteurs commandés ainsi que leurs contraintes tension-courant réelles qui ne sont pas forcément celles d'une phase de la machine. Par exemple, dans le cas d'un couplage étoile des phases, la tension maximale aux bornes des composants est la tension composée aux bornes de la machine, ainsi :

$$
\delta_{\mathrm{g}}^{(i)}=n \cdot k^{(i)} \cdot \delta^{(i)}
$$

où $n$ est le nombre $\cdot$ d'interrupteurs commandés par
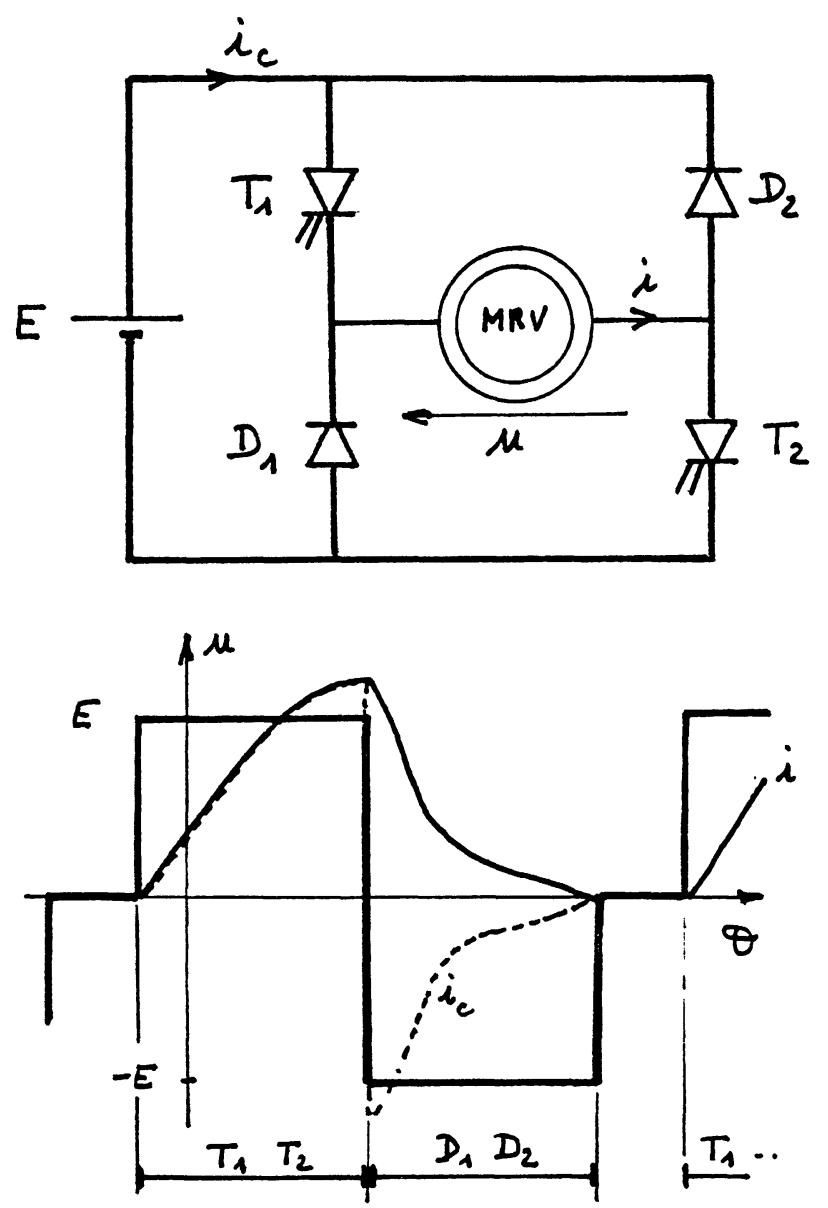

Fig. A1. - Convertisseur d'alimentation en régime non excité.

[Supply convertor in unexcited mode.]
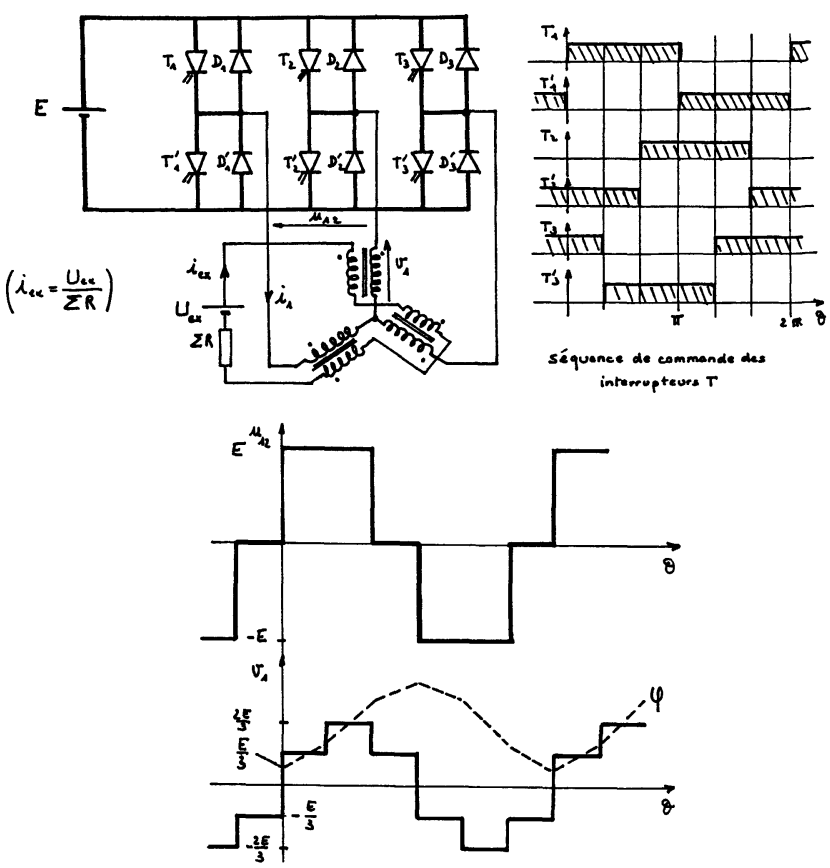

Fig. A2. - Convertisseur d'alimentation en régime excité.

[Supply convertor in excited mode.]

phase et $k^{(i)}$ un coefficient supérieur ou égal à 1 prenant en compte le couplage des phases de la machine et l'alimentation avec une tension dégradée par rapport à la tension optimale (cas de l'alimentation de la figure A2).

Avec le convertisseur en demi-pont (Fig. A1) utilisé en régime non excité, $n=2$ et $k^{(i)}=1$ alors :

$$
\delta_{\mathrm{g}}^{\prime \prime}=2 \cdot \delta^{\prime \prime} \text {. }
$$

Dans le cas de la machine triphasée couplée en étoile et alimentée par la structure de la figure A2 nécessite deux interrupteurs commandés par phase $(n=2)$, la tension maximale aux bornes des composants vaut 1,5 fois la tension simple maximale $\left(k^{\prime \prime}=1,5\right)$. D'autre part le facteur de dimensionnement $\delta_{02}^{\prime \prime}$ est augmenté par rapport à la valeur optimale, conséquence de l'alimentation dégradée pour l'élimination des réactions sur l'excitation.

Un exemple comparatif est donné au tableau A.3. La comparaison porte sur l'alimentation (simulation numérique) de la machine polydiscoïde de $200 \mathrm{~kW}$ (§3.1) par l'une ou l'autre des deux structures précédentes et d'une machine synchrone triphasée couplée en étoile fonctionnant à $\cos \varphi=1$ sous tension sinusoïdale $\left(\delta_{g 0}^{\prime \prime}=2 \sqrt{3} \delta_{0}^{\prime \prime}\right.$ et $\delta_{0}^{\prime \prime}=2$ : voir $\S 2.4)$. Rappelons que les caractéristiques magnétiques de cette machine à réluctance variable ne sont pas optimales. Malgré cela on a obtenu un facteur de dimensionnement global inférieur à celui de la machine synchrone.

On remarque que le régime excité offre un dimensionnement avantageux à condition d'accepter 


\begin{tabular}{|c|c|c|c|}
\hline & $\delta_{g}^{\prime \prime}$ & $\frac{\Delta C}{\langle C\rangle}$ & $\frac{C_{h s}}{\langle C\rangle}$ \\
\hline $\begin{array}{c}\text { MRV non excitée } \\
\text { (fig. A.1) }\end{array}$ & 11,2 & 4,3 & $30 \%$ \\
\hline $\begin{array}{c}\text { MRV excitée mode 1 } \\
\text { (fig. A.2) }\end{array}$ & 6,1 & 10,4 & $12 \%$ \\
\hline Machine synchrone & 7 & & \\
\hline
\end{tabular}

Fig. A3. - Comparaison d'ensembles machines-convertisseurs.

[Comparison of convertor-machine sets.]

une forte pulsation du couple monophasé. En courant unidirectionnel (cas du régime non excité), le courant efficace est plus élevé qu'en courant bidirectionnel pour la même excursion des ampères-tours, dans l'exemple présenté, la machine non excitée nécessite une section de cuivre 2 fois plus élevée que la machine excitée [7] (en prenant en compte l'enroulement d'excitation).

\section{Annexe 2.}

EXPÉRIMENTATION, VALIDATION DES CALCULS. Pour valider cette étude théorique, nous avons réalisé un convertisseur et sa commande d'autopilotage pour alimenter une machine à réluctance variable monophasée d'une centaine de watts. Nous avons mesuré les caractéristiques magnétiques permettant de la modéliser et nous avons effectué différents essais en régime non excité et excité mode 2. Ils nous ont permis de vérifier les calculs numériques. L'hypothèse de Kapp n'étant pas valable, il nous a fallu réaliser un nouveau programme de calcul prenant en compte la résistance du bobinage non négligeable dans cette machine de petite puissance et alimentée avec une très forte densité de courant. Lors des essais, nous avons atteint en particulier, en régime excité, un facteur de dimensionnement global inférieur à 6 à pleine puissance [7].

La structure générale de l'ensemble convertisseurmachine à réluctance variable autopilotée est donnée à la figure A4. La figure A5 montre un exemple des formes d'ondes de tension et courant et du cycle $\varphi(j)$ obtenus en régime non excité $\left(\delta^{\prime \prime}=5\right)$.

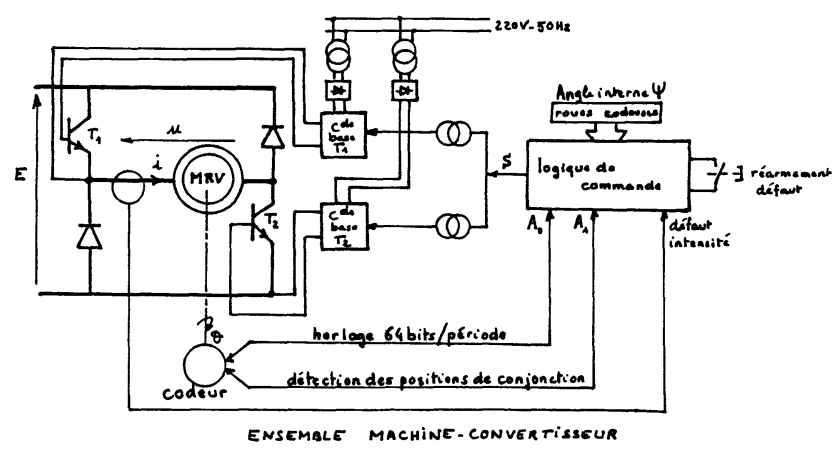

Fig. A4. - Structure générale de la maquette d'essai.

[Structure of the supply and its control.]
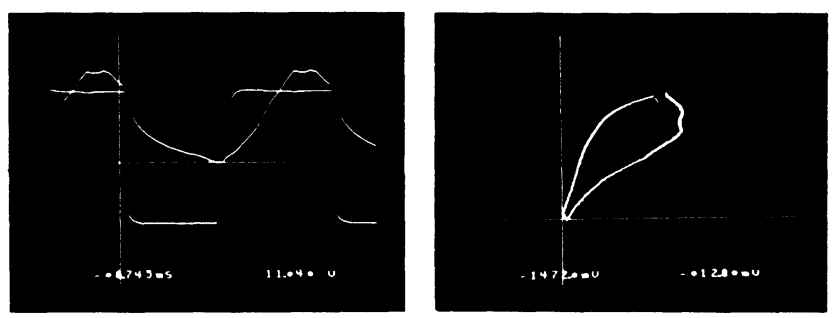

Fig. A5. - Oscillogrammes de la tension, courant et cycle de fonctionnement obtenus en régime non excité $\left(U_{\mathrm{M}}=\right.$ $110 \mathrm{~V}, I_{\mathrm{M}}=2,9 \mathrm{~A}, P=64 \mathrm{~W}, f=148 \mathrm{~Hz}$ ).

[Voltage, current and operating cycle in unexcited mode $\left(U_{\mathrm{M}}=110 \mathrm{~V}, I_{\mathrm{M}}=2.9 \mathrm{~A}, P=64 \mathrm{~W}, f=148 \mathrm{~Hz}\right)$.]

\section{Remerciements.}

Les auteurs tiennent à remercier le Professeur Rioux et le personnel de son laboratoire qui ont rendu possible la réalisation de ce travail et l'ont soutenu financièrement.

\section{Rectificatif.}

Dans l'article référencé [1], les photos sont dues au travail de S. Allano, chercheur C.N.R.S. au Laboratoire d'Electrotechnique à Orsay.

\section{Bibliographie}

[1] Glaize, C., Recherche des formes optimales d'alimentation des machines à réluctance variable, Revue Phys. Appl. 20 (1985) 779 à 794.

[2] Wiart, A., Caussin, G., Marchenoir, A. (Jeumont-Schneider), Machine homopolaire ali- mentée par onduleur de courant pour entraînement de grande puissance à grande vitesse. $R G E$ $93 \mathrm{n}^{\circ} 10$ (1984) 666 à 672.

[3] Meisel, J., Principles of electromechanical energy conversion (McGraw Hill) 1966. 
[4] Faucher, J., Escude, G., Alimentation en courant pour machine à réluctance variable autopilotée. Electron. Appl. Indust. (1979) 47 à 53.

[5] Allano, S., Glaize, C., Reluctance machine supplied with thyristor converter. ICEM (1982) Budapest, p. 579 à 582.

[6] Zhou, J., MAILfert, A., Etude comparative des moteurs associant des aimants permanents et un effet de réluctance variable. Laboratoire EEA Nancy, CNRS, $3^{\mathrm{e}}$ journée d'étude sur les moteurs pas à pas (juin 1984) p. 25 à 33.

[7] Multon, B., Contribution à l'optimisation des ensembles convertisseurs-machines à réluctance variable. Minimisation du Facteur de dimensionnement. Thèse de $3^{\mathrm{e}}$ cycle, Paris VI ( 2 juillet 1985$)$.
[8] Glaize, C., Etude générale et optimisation des modes d'alimentation des machines à réluctance variable. Application aux machines polydiscoïdes. Thèse d'Etat, Paris VI (13 décembre 1983).

[9] Byrne, J. V., McMullin, M. F., A saturable reluctance motor investigated as a spindle in the $\mathrm{kW}$ range. ICEM (1982), Budapest, p. 266 à 269.

[10] Desesquelles, P. F., Contribution à l'étude de l'alimentation des machines à réluctance variable. Simulation numérique de trois ensembles machines-convertisseurs. Thèse de $3^{\mathrm{e}}$ cycle, Paris VI (20 mai 1983).

[11] Allano, S., Glaize, C., Etude systématique de la commande d'une alimentation pulsée pour systèmes de forte puissance. Revue Phys. Appl. 17 (1982) 37 à 44 . 